\title{
32. PETROCHEMISTRY AND GEOCHEMISTRY OF BASALTS IN THE SHIKOKU BASIN AND DAITO BASIN, PHILIPPINE SEA
}

\author{
G. V. Nisterenko, Institute of Geochemistry, Academy of Sciences of the U.S.S.R., Moscow
}

\begin{abstract}
Tholeiitic basalts cored during Deep Sea Drilling Project Leg 58 in the northern Philippine Sea vary in composition: in Hole 443, they correspond to mid-ocean-ridge basalts; in Hole 446A, they correspond to ferrobasalts. There is also a respective increase in degree of differentiation of the parent magma of each type of basalt, with eventual accumulation of iron, titanium, and alkali. The behavior of major and minor elements agrees with an in-chamber magma differentiation. The low magnesium content of the off-ridge basalts in the Shikoku Basin (Hole 442B) is assumed to represent a special case of off-ridge basalts. Ferrobasalts of the Daito Basin clearly indicate that this type of rock occurs extensively among sea-floor basalts. These are also found among continental plateau basalts and as the final products of differentiation of tholeiitic intrusions (ferrogabbros). Hornblende in the ferrobasalts indicates a relatively high water content (as much as 3\%) in the magma. Basalts in Hole 442B do not contain hornblende, which may be attributed to a higher magma temperature.
\end{abstract}

\section{INTRODUCTION}

Drilling into the ocean floor in the northern Philippine Sea has yielded information ample for studying the composition and evolution of a basalt magma within the Shikoku Basin and Daito Basin. A total of 583 meters was drilled in basalts, of which 28.5 meters was drilled in Hole 442A, 163 meters in Hole 442B, 120 meters in Hole 443, 44.5 meters in Hole 444A, 25 meters in Hole 446, and 180 meters in Hole 446A. Holes 442A, 442B, 443 , and $444 \mathrm{~A}$ were drilled in the Shikoku Basin, whereas Holes 446 and 446A were drilled south of the Daito Ridge, which is an ancient remanent arc west of the Kyushu-Palau Ridge.

Approximately 100 samples were studied under the microscope and analyzed by different methods (chemical analysis, X-ray fluorescence, atomic absorption, emission spectroscopy, gamma spectroscopy) for major, minor, and rare elements. A certain degree of rock alteration was recognized, and it was the freshest basalt samples which were selected for further investigation. Petrochemical and geochemical characteristics of the rocks are presented, average basalt compositions are compared, and magma differentiation is considered.

Basalts from Holes 442B, 443, and 446A were regarded as most representative. In addition, information from Holes $444 \mathrm{~A}$ and 446 was used. Basalts from Hole $442 \mathrm{~A}$ are not considered because of intensive alteration.

\section{PETROGRAPHY}

\section{Site $\mathbf{4 4 2}$}

Basalts from Holes 442A and 442B are characterized by high vesicularity (as much as $\mathbf{3 0 \%}$ ), by intensive oxidation, and by calcite deposition, in veinlets and vesicles. The upper 59 meters of the basalts in Hole $442 \mathrm{~B}$ is represented by aphanitic to medium-grained, aphyric varieties. Rock texture is intersertal, intergranular to subophitic. Less common (Hole 442B) are sparsely phyric basalts with phenocrysts of plagioclase and extremely rare clinopyroxene. Two basalts have spherulitic texture, with randomly distributed plagioclase spherulites in a cryptocrystalline groundmass with plagioclase and clinopyroxene microlites.

The lower units of Hole 442B ( $102 \mathrm{~m}$ below a sedimentary-rock interlayer) are pillow basalts, which are actually cobbles with a glass rind up to $10 \mathrm{~mm}$ thick. The internal parts of the pillow basalts are rocks with intersertal and, less commonly, intergranular texture. A variolitic texture, 1 to $2 \mathrm{~mm}$ thick, which is typical of pillow basalts, is formed at the contact with the glass rind.

Plagioclase $\left(\mathrm{An}_{60-70}\right)$ and clinopyroxene (augite) are the principal rock-forming minerals of the basalts in Hole 442B. There is no olivine, and ore minerals are subordinate.

\section{Site $\mathbf{4 4 3}$}

Lava flows from Hole 443 are primarily phyric basalts, with subordinate pillow basalts. The latter are confined to the lowermost part of the section, where they are intercalated with the lava flows (Site 443 report, this volume). Vesicularity of the flows is 0 to 5 per cent; it reaches 30 per cent in the pillow basalts. The vesicles are filled by calcite, smectite, and occasionally by wellformed pyrite octahedrons. 
The rocks have a fine- to medium-grained texture, with plagioclase phenocrysts $\left(\mathrm{An}_{60}\right.$; as much as $\left.20 \%\right)$ and, less commonly, olivine $\left(\mathrm{Fo}_{75-85}\right)$ phenocrysts.

The groundmass texture varies from cryptocrystalline and intersertal in flow contact zones to intergranular and subophitic in the central parts of flows, which are mostly well crystallized. The groundmass is composed of plagioclase $(30-50 \%)$, clinopyroxene $(20-45 \%)$, olivine $(0-15 \%)$ and ore minerals $(2-7 \%)$. Depending on basalt texture, glass content varies from 5 or 10 per cent to 90 per cent. Olivine is essentially replaced by an aggregate of serpentine and talc; glass is palagonitized.

The thickest flows exhibit a melt differentiation, that is, olivine content increases toward the flow bottom.

The pillow basalts, compared to those of Hole 442B, have a more-devitrified glass rind. Their internal parts are composed of rocks with intersertal and intergranular textures. In mineral composition, these rocks are similar to the flow basalts.

\section{Site 444}

Hole 444A penetrated a sill, 12 meters thick, separated from underlying basalts by a 20 -meter sequence of sedimentary rocks. The sill is aphyric and mediumgrained, with a texture ranging from intersertal at the contacts to intergranular and diabasic in the central parts. The rock consists of plagioclase $\left(\mathrm{An}_{60-80} ; 30 \%\right)$, augite $(20-25 \%)$, olivine $(1-15 \%)$, magnetite $(2-3 \%)$, and groundmass $(30-45 \%)$ which contains chlorite, clays, and actinolite. Olivine content increases from top to bottom (from 1 to $15 \%$ ), which indicates magma differentiation in the process of sill formation.

The basalts below the 20 -meter unit of sedimentary rocks are plagioclase phyric varieties with a plagioclase phenocryst content $\left(\mathrm{An}_{60}\right)$ of 2 to 30 per cent. The basalts have intergranular to diabasic texture and are composed of plagioclase $\left(\mathrm{An}_{70}, 30-50 \%\right)$, augite $(25-$ $30 \%)$, olivine $(1-10 \%)$, and magnetite $(1-5 \%)$; the groundmass is composed of a fine-grained aggregate of chlorite, clay, and (apparently) actinolite.

\section{Site 446}

Basalts in Holes 446 and $446 \mathrm{~A}$ occur as sills 0.3 to 22 meters thick in the sedimentary-rock sequence; they were encountered at sub-bottom depths of 384 to 628.5 meters. These basalts are massive and are characterized by chilled zones both at the top and bottom, fine- to medium-grained texture, and generally low vesicularity $(0-10 \%$, on the average $3 \%)$. However, in a number of cases vesicularity increases to 15 to 30 per cent (Site 446 report, this volume).

The basalts are essentially aphyric, with intersertal and intergranular texture. They contain as much as 35 per cent plagioclase $\left(\mathrm{An}_{60-80}\right), 25$ per cent clinopyroxene, and 20 per cent titanomagnetite. Basalts with a high vesicularity $(15-30 \%)$ contain as much as 15 per cent hornblende, which is confined to well-formed grains in the groundmass (glass), filling spaces between clinopyroxene grains. This may be vividly exemplified by basalts of Section 446-13-1, $17 \mathrm{~cm}$, where the slightly devitrified groundmass $(60 \%)$ contains only large crystals of titaniferous clinopyroxene and smaller, but well-formed hornblende grains. This, and the magmatic nature of the amphibole, points to crystallization of magma with the removal of pyroxene and, subsequently, hornblende from the melt, evidently without crystallization of plagioclase in the early stages of the process.

Olivine has not been found in Hole 446A basalts. However, a number of sills apparently contain pseudomorphs of secondary minerals after olivine.

\section{ORE MINERALS}

The most common ore minerals in the basalts are titanomagnetite, pyrite, chalcopyrite, and native copper.

Titanomagnetite forms irregular, platy, skeletal and dendritic grains. The latter occur primarily in the basalts of Hole 446A. The mineral is generally single-phase, without exsolution structures. However, there are occasional two-phase grains with titanomagnetite in the center and magnetite at the periphery (Section 442B-6-3, $27 \mathrm{~cm}$ ). In some cases, zoned and polysynthetic aggregates are found (Section 442B-8-1, $80 \mathrm{~cm}$ ). In addition, the basalts have some magnetite impregnated in silicates.

Sulfides (pyrite, chalcopyrite) are represented by irregular grains and droplets. The grains are confined to interstices and, in certain cases (pyrite), to vesicles, where they may have considerable size (as much as 1.5-2 $\mathrm{mm}$ ). Chalcopyrite is occasionally crystallized along with titanomagnetite. Almost all the basalts contain drop-like sulfide inclusions, indicating immiscibility of sulfide and silicate melts. These are found both in interstices and within rock-forming minerals (for example, in plagioclase).

Native copper commonly occurs as fine, reddish grains and is apparently a secondary mineral formed by sulfide oxidation and redeposition of copper by solutions forming carbonate veins in the basalts.

\section{METHODOLOGICAL PROBLEMS}

To arrive at reliable petrochemical and geochemical conclusions, particular attention was paid to analysis quality. For this purpose, 18 of 30 elements were analyzed by two or even three independent methods. For example, major elements were analyzed by X-ray fluorescence and ( $60 \%$ of the samples) by chemical methods. Copper, nickel, cobalt, vanadium, and strontium were analyzed by atomic absorption and spectrochemical methods. Phosphorus and chromium were analyzed by atomic absorption, X-ray fluorescence, and spectrochemical methods. Comparison of the obtained data has shown good agreement.

For petrochemical analysis, we have primarily utilized chemical data, with subordinate $(40 \%)$ application of the X-ray-fluorescence results.

Rock alteration and sample representativeness are additional principal problems.

Almost all the oceanic basalts have been subjected to various degrees of reaction with sea water, hydrothermal alteration, and metamorphism. The total effect of these superimposed processes may be considerable, sometimes greatly changing the initial chemical compo- 
sition of rocks. For instance, even low-temperature interaction of sea water and pillow basalts within a distance of $2 \mathrm{~cm}$ from the chilled zone represented by a glass rind results in removal of more than 1 per cent magnesium and in a doubling of the potassium content (Table 1, sample from Hole 442B; see also Nisterenko et al., this volume).

Sometimes even more conspicuous are the intensively altered internal parts of pillow basalts, where addition of potassium is also observed (Table 1 , sample from Hole 443). Iron is strongly oxidized in this process. The intensive removal of silica from basalts, previously noted by Hart (1976), is not clearly evident in these rocks, which are slightly bleached near the chilled (glass) zone and considerably altered (gray-yellow color) in the internal parts of the pillow basalts.

Alteration results from interaction of the basalts with sea water, formation of palagonite, and replacement by low-temperature secondary minerals such as smectite and zeolites with high potassium and low magnesium content (Frey et al., 1974). An increased calcium content is accounted for by deposition of calcite in veinlets and vesicles. No interrelation between element distribution and water content, which may be a rock-alteration index, has been ascertained for Shikoku and Daito basalts (Hart, 1976).

There is a close interdependence between calcium and carbon-dioxide concentration (Figure 1). This apparently points not only to calcite deposition in the rocks whose content increases proportional to carbon dioxide concentration, but also, as is shown in Table 1, to an apparent supply of alkali to the rock and magnesium removal.

Norms of these basalts show nepheline., i.e., they shift to the alkali zone. As shown in Figure 1, we have taken 0.3 per cent carbon-dioxide concentration as the upper limit for unaltered (slightly altered) basalts. All the analyses for Hole 442A and some of those for other holes (see appendix) are thus excluded.

Also important is sample representativeness. This problem warrants particular investigation, because

TABLE 1

Chemical Compositions of Fresh Basaltic Glass and Altered Pillow Basalt

\begin{tabular}{|c|c|c|c|c|c|c|c|}
\hline \multirow[b]{3}{*}{$\begin{array}{l}\text { Major } \\
\text { Oxides } \\
(\%)\end{array}$} & \multicolumn{3}{|c|}{ Sample 442B-10-1, 118-121 cm } & \multicolumn{2}{|c|}{$\begin{array}{c}\text { Sample } 443-57-2, \\
\text { I } 05-108 \mathrm{~cm}\end{array}$} & \multicolumn{2}{|c|}{$\begin{array}{c}\text { Sample 443-57-2, } \\
114-118 \mathrm{~cm}\end{array}$} \\
\hline & $\begin{array}{c}\text { Glass Rind } \\
\text { of } \\
\text { Pillow } \\
\text { Basilt }\end{array}$ & \multicolumn{2}{|c|}{$\begin{array}{l}\text { Pillow Basalt } \\
\text { (i-2 cm from } \\
\text { glass rind) }\end{array}$} & \multicolumn{2}{|c|}{$\begin{array}{l}\text { Glass Rind of } \\
\text { Pillow Basalt }\end{array}$} & \multicolumn{2}{|c|}{$\begin{array}{l}\text { Inner part of Pillow } \\
\text { Basalt }(10 \mathrm{~cm} \text { from } \\
\text { glass rind })\end{array}$} \\
\hline & \multicolumn{2}{|c|}{$X$-ray fluorescence } & $\begin{array}{c}\text { Chem- } \\
\text { ical }\end{array}$ & $\begin{array}{l}\text { X-ray } \\
\text { fluo- } \\
\text { rescence }\end{array}$ & $\begin{array}{l}\text { Chem- } \\
\text { ical }\end{array}$ & $\begin{array}{l}\text { X-ray } \\
\text { fluo- } \\
\text { rescence }\end{array}$ & $\begin{array}{l}\text { Chem- } \\
\text { ical }\end{array}$ \\
\hline $\mathrm{SiO}_{2}$ & 50.20 & 49.20 & 48.40 & 49.60 & 48.70 & 49.90 & 47.10 \\
\hline $\mathrm{TiO}_{2}^{2}$ & 1.63 & 1.59 & 1.62 & 1.77 & 1.84 & 1.82 & 1.93 \\
\hline $\mathrm{Al}_{2} \mathrm{O}_{3}$ & 15.64 & 15.36 & 15.25 & 15.36 & 14.50 & 14.61 & 14.60 \\
\hline $\mathrm{Fe}_{2} \mathrm{O}_{3}$ & 10.05 & 10.79 & $\begin{array}{l}7.22 \\
4.12\end{array}$ & 10.63 & $\begin{array}{l}1.95 \\
9.32\end{array}$ & 11.96 & $\begin{array}{l}6.34 \\
5.55\end{array}$ \\
\hline $\begin{array}{l}\mathrm{FeO} \\
\mathrm{MnO}\end{array}$ & - & 8 & $\begin{array}{l}4.12 \\
0.18\end{array}$ & ? & $\begin{array}{l}9.32 \\
0.19\end{array}$ & 8 & $\begin{array}{l}5.55 \\
0.22\end{array}$ \\
\hline $\mathrm{MgO}$ & & & 5.63 & 7.65 & 7.60 & 5.75 & 5.86 \\
\hline $\mathrm{CaO}$ & 11.87 & 13.10 & 11.94 & 10.26 & 10.25 & 12.20 & 11.70 \\
\hline $\mathrm{N}_{2} \mathrm{O}$ & & 3.11 & 3.20 & 2.60 & 2.70 & 2.86 & 2.88 \\
\hline $\mathrm{K}_{2} \mathrm{O}$ & 0.20 & 0.48 & 0.40 & 0.32 & 0.28 & 0.68 & 0.56 \\
\hline $\mathrm{CO}_{2}$ & - & 0.17 & 0.17 & 0.60 & 0.62 & 1.03 & 0.70 \\
\hline $\mathrm{H}_{2} \mathrm{O}+$ & - & - & 1.75 & - & 0.91 & - & 0.79 \\
\hline Total & 99.60 & 99.90 & 100.54 & 98.60 & 99.32 & 100.20 & 99.40 \\
\hline
\end{tabular}

there is no compositional homogeneity (or else homogeneity is extraordinary); thus, random sampling may not reflect average composition. This is clear from Figure 2. An individual sample may only fortuitously reflect the average composition of a lava flow. In view of this fact, we have taken, wherever possible, average values of several determinations for a flow. Data for the leastaltered samples (carbon-dioxide concentration below $0.3 \%$ ) and averages for individual flows are given in Table 2. Analyses of unrepresentative samples are given in an appendix; these represent basalts with high carbon-dioxide concentration and extensive oxidation.

\section{PETROCHEMISTRY}

Table 2 and Figures 3 and 4 show that almost all basalts in the Shikoku Basin and Daito Basin are tholeiitic (Irvine and Baragar, 1971). Only the upper lava flow of Hole $444 \mathrm{~A}$ and two samples of Site 446 are in the alkali fields of Figures 3 and 4 . According to normative composition (Table 2), basalts of Hole 443 are olivine tholeiites; those of Hole 442B are saturated tholeiites (normative hypersthene); and those of Site 446 are oversaturated tholeiites (normative quartz and hypersthene) (Yoder and Tilley, 1962).

However, basalts of Sites 442 and 446 should actually be more basic. Their high acidity with respect to normative composition may be accounted for by substantial oxidation of iron by low-temperature reactions with sea water. Therefore, ferric oxide equals or exceeds ferrous oxide. Hence, the norm shows exceptionally high, unnatural concentrations of magnetite (6-10\%), without olivine. This is exemplified by basalts of Hole 443. The first three samples listed for this hole in Table 2 have a nearly normal $\mathrm{Fe}_{2} \mathrm{O}_{3} / \mathrm{FeO}$ ratio, and they show normative olivine. Rocks of the same hole with a higher degree of oxidation of iron show no normative olivine. We should, therefore, believe that the lava flows of Hole 442B are actually olivine tholeiites, as are the typical pillow basalts of the same hole (Table 2 ). They differ greatly from the basalts of Hole 443 in a lower content of normative olivine for most of the rocks (Figure 5).

This also applies to basalts of Site 446, which apparently correspond not to oversaturated but to rather saturated tholeiites.

Comparison of average chemical compositions of basalts of these three holes with typical oceanic-profile samples (TOPS) and mid-ocean-ridge basalt (MORB) (Table 3 ) shows that the Hole 443 rocks correspond to MORB although they have somewhat lower iron, chromium, and nickel contents.

The basalts of Holes 442B and 446A have somewhat lower contents of magnesium, chromium, and nickel than does MORB, and they have somewhat higher contents of iron, potassium, strontium, and rubidium. The $\mathrm{K} / \mathrm{Na}$ and $\mathrm{Ti} / \mathrm{Cr}$ ratios are also higher for basalts of Holes 442B and 446A than for MORB.

Some comparisons among Leg 58 basalts are shown in Figure 6.

The basalts of Hole 442B are distinct from MORB not only in the lava flows making up the upper units, but also in the fresh glass of the pillow basalt in the 


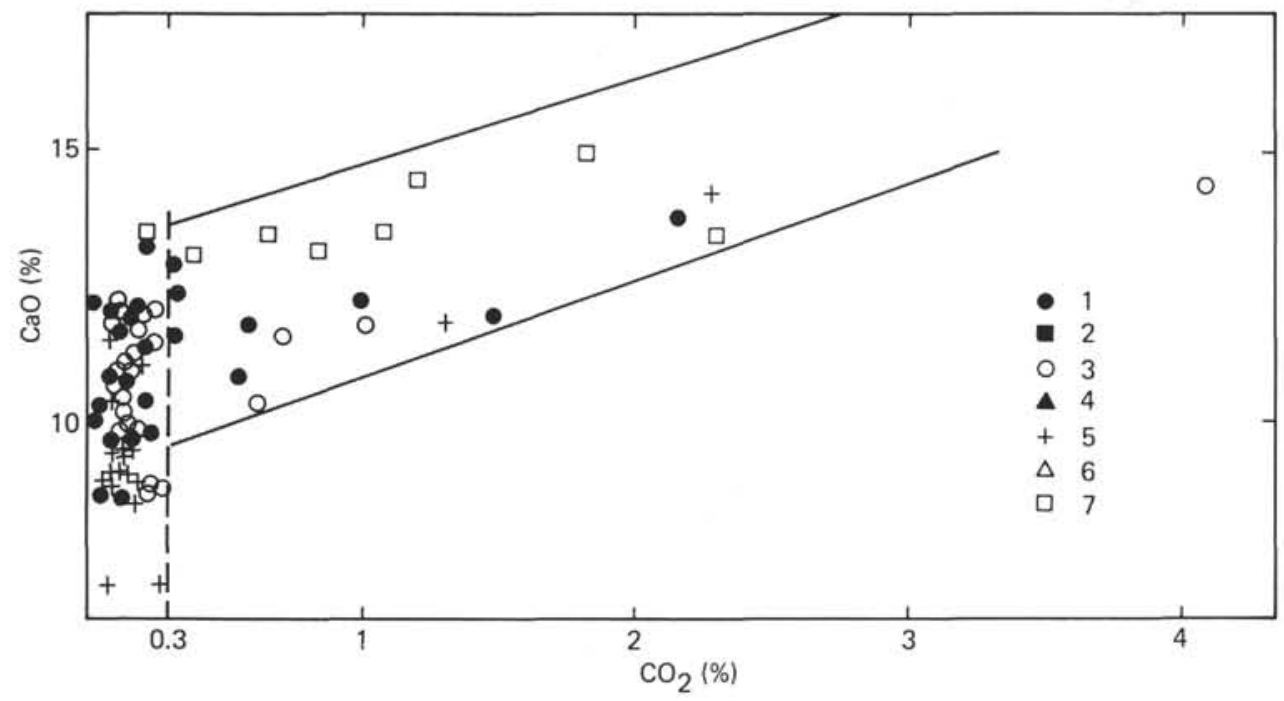

Figure 1. Relationship between calcium and carbon-dioxide contents in basalt. Rocks with carbon-dioxide content exceeding 0.3 per cent (dashed line) are considered strongly altered. Here, and in other figures, the numbers refer to: (1) Hole $442 B$ basalts; (2) glass in Hole 442B pillow basalts; (3) Hole 443 basalts (and two lava flows in Hole 444A; Table 2); (4) glass in Hole 443 pillow basalts; (5) Hole $446 \mathrm{~A}$ basalts; (6) Hole $444 \mathrm{~A}$ alkaline basalt sill; (7) Hole $442 \mathrm{~A}$ basalts.

Samples

$\mathrm{SiO}_{2} \quad \mathrm{TiO}_{2} \quad \mathrm{Al}_{2} \mathrm{O}_{3} \quad \Sigma \mathrm{Fe}(\mathrm{FeO})$

$\mathrm{MgO}$

$\mathrm{CaO}$

$\mathrm{Na}_{2} \mathrm{O} \quad \mathrm{K}_{2} \mathrm{O}$

\begin{tabular}{llllllllllllllllllllllllllllllll}
47 & 48 & 49 & 50 & 1 & 2 & 14 & 15 & 16 & 17 & 7 & 8 & 9 & 10 & 5 & 6 & 7 & 8 & 9 & 10 & 8 & 9 & 10 & 11 & 12 & 2 & 3 & 4 & 0 & 1 & 2 & $\%$ \\
\hline
\end{tabular}

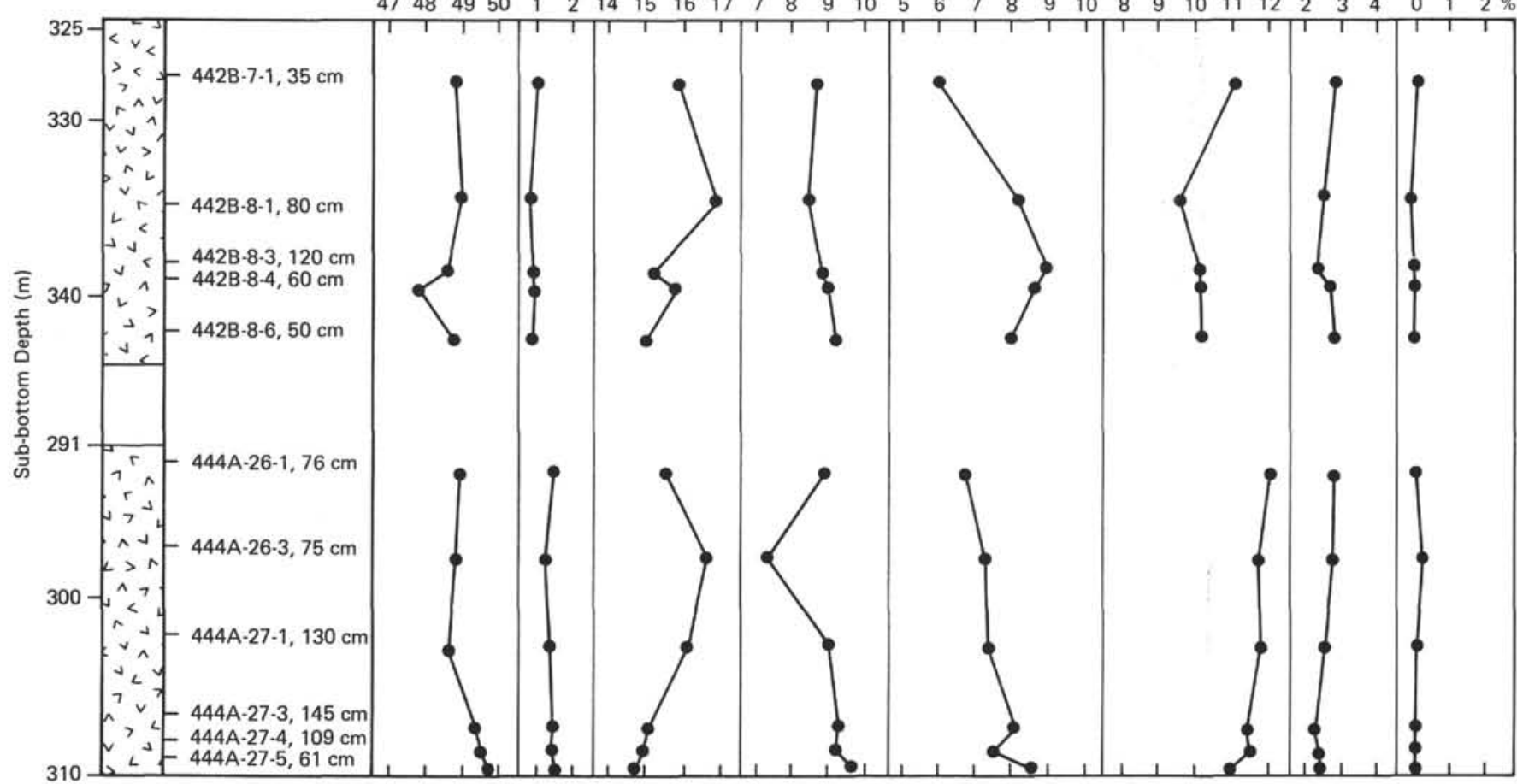

Figure 2. Vertical variations in chemical composition of basalt flows.

lower part of the hole (Tables 2 and 3). Because the Hole 442B basalts are of off-ridge origin (Klein et al., 1978), their lower magnesium content is natural. The data presented by Hart (1976) on the composition of
TOPS suggest that the chemical features of Hole 442B basalts probably are typical of off-ridge basalts.

Thus, a lower magnesium content is seemingly characteristic of ocean-floor and marginal-sea basalts which 
TABLE 2

Chemical Composition and Norms of the Freshest Basalts, Shikoku Basin and Daito Ridge

\begin{tabular}{|c|c|c|c|c|c|c|c|c|c|c|c|}
\hline Sample (interval in $\mathrm{cm}$ ) & $\mathrm{SiO}_{2}$ & $\mathrm{TiO}_{2}$ & $\mathrm{Al}_{2} \mathrm{O}_{3}$ & $\mathrm{Fe}_{2} \mathrm{O}_{3}$ & $\mathrm{FeO}$ & $\mathrm{MnO}$ & $\mathrm{MgO}$ & $\mathrm{CaO}$ & $\mathrm{Na}_{2} \mathrm{O}$ & $\mathrm{K}_{2} \mathrm{O}$ & $\mathrm{P}_{2} \mathrm{O}_{5}$ \\
\hline $442 \mathrm{~B}-3-3,140-146$ & 49.61 & 1.26 & 15.80 & 6.15 & 2.89 & 0.13 & 6.90 & 11.63 & 2.71 & 0.33 & 0.114 \\
\hline $4-2,35-40$ & 49.20 & 1.23 & 16.35 & 5.72 & 2.95 & 0.11 & 6.41 & 12.33 & 3.06 & 0.34 & 0.119 \\
\hline $5 \cdot 2,65-70$ & 49.60 & 1.22 & 16.25 & 4.04 & 4.24 & 0.11 & 7.78 & 10.65 & 3.10 & 0.20 & 0.098 \\
\hline $6-3,27-35$ & 49.40 & 1.81 & 15.00 & 2.43 & 8.70 & 0.19 & 7.38 & 10.25 & 2.58 & 0.31 & 0.039 \\
\hline $7-1,35-42$ & 49.00 & 1.26 & 16.20 & 6.01 & 3.50 & 0.15 & 6.20 & 11.30 & 3.06 & 0.19 & \\
\hline $8-1,80-85$ & 49.20 & 1.12 & 17.20 & 4.07 & 4.98 & 0.14 & 8.40 & 8.60 & 2.73 & 0.13 & 0.140 \\
\hline $8 \cdot 3,120-125$ & 48.40 & 1.17 & 15.40 & 3.21 & 6.11 & 0.15 & 9.13 & 9.60 & & 0.15 & 0.080 \\
\hline $8 \cdot 4,60-65$ & 48.00 & 1.21 & 16.05 & 3.48 & 6.05 & 0.13 & 8.80 & 9.65 & 2.92 & 0.18 & 0.080 \\
\hline $8-6,50-55$ & 49.00 & 1.16 & 15.20 & 4.73 & 5.10 & 0.12 & 8.25 & 9.70 & 3.02 & 0.16 & 0.107 \\
\hline (Avg., $7-1$ to $8-6$ ) & 48.72 & 1.18 & 16.00 & 4.30 & 5.15 & 0.14 & 8.16 & 9.77 & 2.87 & 0.16 & 0.116 \\
\hline $8 \cdot 6,135-140$ & 49.50 & 1.26 & 16.10 & 5.42 & 3.76 & 0.10 & 7.60 & 10.00 & 3.12 & 0.16 & 0.052 \\
\hline $8 \cdot 7,47-52$ & 48.60 & 1.19 & 15.10 & 6.76 & 3.32 & 0.14 & 8.50 & 8.55 & & 0.35 & \\
\hline (Avg., 8-6 and 8.7) & 49.05 & 1.22 & 15.60 & 6.09 & 3.54 & 0.12 & 8.05 & 9.27 & 3.26 & 0.25 & 0.046 \\
\hline $9-1,10-15$ & 49.80 & 1.03 & 16.45 & 5.53 & 2.53 & 0.15 & 8.03 & 10.26 & 2.71 & 0.27 & \\
\hline $10-1,78-84$ & 49.00 & 1.60 & 16.10 & 7.00 & 4.77 & 0.19 & 5.40 & 10.80 & 3.04 & 0.39 & 0.169 \\
\hline $10-1,93-96^{a}$ & 48.10 & 1.61 & 13.75 & 9.7 & & 0.16 & 6.19 & 13.15 & 3.12 & 0.34 & 0.119 \\
\hline $\begin{array}{l}\text { (Avg., upper } 59 \mathrm{~m} \text {. of } \\
\text { the hole) }\end{array}$ & 49.16 & 1.35 & 15.70 & 5.16 & 4.35 & 0.12 & 7.14 & 10.90 & 2.94 & 0.29 & 0.103 \\
\hline $10-1,118-121^{b}$ & 48.40 & 1.63 & 15.25 & 7.22 & 4.12 & 0.18 & 5.63 & 11.94 & 3.20 & 0.40 & 0.140 \\
\hline $14-1,35-40 a, b$ & 50.77 & 1.67 & 16.27 & 9.1 & & - & 7.15 & 11.83 & 2.87 & 0.21 & - \\
\hline $14-1,85-90^{\mathrm{a}, \mathrm{b}}$ & 51.07 & 1.56 & 16.09 & 9.2 & & - & 7.14 & 11.67 & 2.98 & 0.25 & - \\
\hline $14-1,115-120^{a}, b$ & 50.91 & 1.51 & 16.24 & 9.12 & & - & 7.23 & 11.71 & & 0.24 & - \\
\hline $16-1,35-47$ & 49.40 & 1.33 & 16.80 & 5.23 & 4.74 & 0.16 & 5.54 & 12.00 & 2.87 & 0.40 & 0.150 \\
\hline $17-1,50-55$ & 49.60 & 1.74 & 16.10 & 5.58 & 4.83 & 0.17 & 5.33 & 11.86 & 3.20 & 0.40 & 0.203 \\
\hline $17-1,84-86^{a}, b$ & 51.54 & 1.63 & 15.60 & 9.9 & & - & 6.50 & 11.56 & 2.98 & 0.25 & - \\
\hline $17-1,117-120 a, b$ & 51.52 & 1.64 & 15.55 & 10.0 & & - & 6.51 & 11.45 & 2. & 0.27 & - \\
\hline $17 \cdot 2,10-15 \mathrm{a}, \mathrm{b}$ & 51.54 & 1.59 & 15.35 & 10.01 & & - & 6.80 & 11.81 & 2.61 & 0.24 & - \\
\hline $19 \cdot 2,65-70^{\mathrm{a}, \mathrm{b}}$ & 51.90 & 1.65 & 15.70 & 9,8 & & - & 6.56 & 11.49 & 2.52 & 0.24 & - \\
\hline $20-1,57-61^{a}, b$ & 51.72 & 1.64 & 15.66 & $9.8 \mathrm{C}$ & & - & 6.86 & 11.64 & 2,38 & 0.25 & - \\
\hline $\begin{array}{l}\text { (Avg., lower } 102 \mathrm{~m} \text { of } \\
\text { the hole) }\end{array}$ & 50.76 & 1.60 & 15.90 & 9.7 & & - & 6.49 & 11.74 & 2.87 & 0.29 & - \\
\hline (Avg., Hole 442B) & 49.72 & 1.49 & 15.81 & 9.35 & & - & 6.78 & 11.36 & 2.91 & 0.29 & - \\
\hline $443-53-1,15-20$ & 48.60 & 1.48 & 16.70 & 2.92 & 7.37 & 0.16 & 7.65 & 11.00 & 2.80 & 0.11 & 0.116 \\
\hline $54-4,115-120$ & 48.60 & 1.38 & 16.20 & 2.88 & 7.22 & 0.16 & 8.30 & 10.95 & 2.77 & 0.14 & 0.109 \\
\hline $54-7,63-67$ & 48.40 & 1.46 & 15.60 & 2.81 & 7.55 & 0.16 & 8.15 & 11.40 & 2. & 0.07 & 0.103 \\
\hline $57-2,105-108 a, b$ & 49.88 & 1.77 & 16.11 & 10.07 & & - & 7.52 & 11.84 & 2.49 & 0.28 & - \\
\hline $57 \cdot 3,0-5$ & 48.80 & 1.24 & 16.10 & 4.10 & 4.59 & 0.11 & 8.03 & 10.10 & 3.05 & 0.23 & 0.189 \\
\hline $58-2,71-75$ & 48.80 & 1.78 & 15.40 & 6.01 & 4.94 & 0.11 & 6.73 & 9.80 & 3.24 & 0.53 & 0.178 \\
\hline $58-2,125-128$ & 49.20 & 1.70 & 16.35 & 5.29 & 4.05 & 0.10 & 7.08 & 9.95 & 3.12 & 0.36 & 5 \\
\hline $58-3,121-125$ & 49.20 & 1.88 & 15.30 & 4.16 & 5.72 & 0.11 & 7.82 & 9.77 & 3.08 & 0.30 & 3 \\
\hline (Avg., 58-2 to 58-3) & 49.07 & 1.79 & 15.68 & 5.15 & 4.90 & 0.11 & 7.17 & 9.64 & & 0.39 & 0.172 \\
\hline $61-1,106-110$ & 48.20 & 1.18 & 16.20 & 4.38 & 4.64 & 0.13 & 9.17 & 10.70 & 2.62 & 0.09 & .096 \\
\hline $62-1,88-93$ & 49.60 & 1.32 & 16.20 & 3.07 & 4.86 & 0.12 & 7.73 & 11.97 & 2.77 & 0.11 & \\
\hline $63-8,89-94$ & 49.00 & 1.32 & 16.20 & 5.34 & 3.69 & 0.13 & 7.86 & 11.21 & 2.92 & 0.10 & 3 \\
\hline $64-2,84-86$ & 48.00 & 1.26 & 16.20 & 6.48 & 2.34 & 0.15 & 8.28 & 10.85 & 278 & 0.09 & 6 \\
\hline (Avg., 63-8 and 64-2) & 48.50 & 1.29 & 16.20 & 5.91 & 3.01 & 0.14 & 8.07 & 11.03 & 2.85 & 0.09 & 0.100 \\
\hline (Avg., Hole 443) & 48.72 & 1.39 & 16.11 & 4.31 & 5.18 & 0.14 & 8.03 & 10.85 & 2.83 & 0.15 & 0.120 \\
\hline $444 \mathrm{~A}-20-1,39-43^{\mathrm{C}}$ & 49.70 & 2.18 & 15.32 & 8.18 & & 0.15 & 5.78 & 8.66 & 3.88 & 2.61 & 0.140 \\
\hline $20-1,77-81 \mathrm{c}$ & 47.30 & 2.08 & 15.60 & 3.83 & 4.28 & 0.16 & 5.42 & 10.06 & 3.9 & 2.39 & \\
\hline $20-4,41-45^{c}$ & 46.50 & 1.50 & 16.60 & 3.29 & 4.85 & 0.14 & 9.02 & 7.35 & 3.45 & 1.90 & 0.090 \\
\hline (Avg., 20-1 to 20-4) d & 47.83 & 1.92 & 15.84 & 3.56 & 4.56 & 0.15 & 6.74 & 8.69 & 3.74 & 2.30 & 0.142 \\
\hline $25 \cdot 3,111-115$ & 49.20 & 1.53 & 17.12 & 4.13 & 5.20 & 0.15 & 6.79 & 11.85 & 2.79 & 0.20 & 0.146 \\
\hline $26-1,76-80$ & 49.20 & 1.50 & 16.40 & 3.80 & 5.40 & 0.16 & 6.90 & 12.43 & 2.81 & 0.24 & 4 \\
\hline $26-3,75-79 a$ & 48.80 & 1.27 & 16.86 & 7.51 & & 0.17 & 7.48 & 11.92 & & 0 & 4 \\
\hline $27-1,131-135$ a & 48.50 & 1.39 & 16.30 & 9.20 & & 0.16 & 7.49 & 12.00 & 2 . & 0.32 & .160 \\
\hline $27-3,145-150^{a}$ & 49.30 & 1.43 & 15.18 & 9.43 & & 0.16 & 8.23 & 11.63 & 2.52 & 0.30 & 6 \\
\hline $27-4,119-123 a$ & 49.40 & 1.46 & 15.03 & 9.41 & & 0.16 & 7.68 & 11.70 & 2.64 & 0.29 & .162 \\
\hline $27-5,61-65$ & 50.70 & 1.50 & 14.87 & 3.61 & 5.88 & 0.18 & 6.95 & 10.41 & 2.72 & 0.23 & 74 \\
\hline (Avg., 26-1 to 27-5) d & 49.32 & 1.42 & 15.92 & 3.70 & 5.64 & 0.16 & 7.45 & 11.68 & 2.73 & 0.29 & 0.153 \\
\hline $46 \mathrm{~A}-5$ & 0 & 3. & 1 & 4.44 & 9.60 & 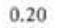 & Ji & 9. & 2 & & \\
\hline $8-2,144-150$ & 47.00 & 4.10 & 13.50 & 5.58 & 10.09 & 0.24 & 5.42 & 8.90 & 2.4 & 0 . & 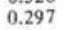 \\
\hline $9 \cdot 3,90-95$ & 47.60 & 4.20 & 13.14 & 6.50 & 8.65 & 0.23 & 5.50 & 8.85 & 2.6 & 5 & 0 \\
\hline $10-5,97-102$ & 44.00 & 5.40 & 14.0 & 5.23 & 7.23 & 0.21 & 6.00 & 11.50 & & & \\
\hline $14-3,97-102^{\mathrm{a}}$ & 50.30 & 3.67 & 13.13 & 12.52 & & 0.16 & 6.54 & 10.15 & 2. & 0.32 & 4 \\
\hline $15-4,38-43$ & 49.40 & 3.80 & 13.20 & 6.55 & 6.60 & 0.15 & 5.87 & 8.97 & 2. & 0. & \\
\hline $17, \mathrm{CC}, 24-32$ & 46.20 & 4.40 & 15.20 & 6.83 & 6.46 & 0.27 & 4.8 & 9.45 & 2.6 & 1.26 & 8 \\
\hline $19-3,0-8$ & 48.20 & 3.90 & 14.00 & 5.1 & 7.99 & 0.20 & 6.1 & 8.93 & 3.2 & 0.38 & \\
\hline $19-3,67-73$ & .00 & 4.20 & 13.80 & 5.49 & 8.60 & 0.24 & 5.07 & 9.50 & 2 & & \\
\hline (Avg, 19-3) & 48.10 & 4.05 & 13.90 & 5.31 & 8.29 & 0.22 & 5.59 & 9.21 & 2.94 & 0.37 & 0.377 \\
\hline $21-2,82-88 a$ & 49.30 & 3.88 & 12.95 & 13.31 & & 0.16 & 5.98 & 11.12 & 2.30 & 0.12 & 0. \\
\hline $21-5,140-146^{a}$ & 50.60 & 4.02 & 12.06 & 13.63 & & 0.17 & 5.11 & 9.91 & 2.72 & 0.76 & \\
\hline $21-6,53-58$ & 51.60 & 4.10 & 12.90 & 6.02 & 7.82 & 0.20 & 4.87 & 7.00 & 2. & & \\
\hline (Avg., 21-2 to $21-6)^{d}$ & 50.50 & 4.00 & 12.64 & 6.02 & 7.82 & 0.18 & 5.32 & 9.34 & & 0.53 & \\
\hline $21-6,69-74$ & 47.40 & 4.10 & 14.7 & 5.47 & 8.57 & 0.25 & 5.2 & 8.87 & & 0. & 6 \\
\hline $22 \cdot 1,132-137$ & 46.00 & 5.04 & 14.35 & 8.60 & 6.76 & 0.28 & 5.41 & 7.00 & 3.25 & & \\
\hline $22-4,0-6^{a}$ & 46.90 & 4.61 & 12.45 & 15.34 & & 0.22 & 5.56 & 11.08 & 2.48 & 0.22 & 2 \\
\hline Avg., $22-1$ and $22-4)^{d}$ & 4 & 4. & 13.4 & 8.60 & 6. & 0.25 & 5.48 & 9.04 & & & \\
\hline $23-6,78-8$ & 48.10 & 3.62 & 14.25 & 6.25 & 6.29 & 0.17 & 6.8 & 9.59 & & & \\
\hline $24-3,78-85 \mathrm{a}$ & 52.20 & 3.50 & 12.52 & 11.62 & & 0.22 & 4.64 & 11.28 & 2. & & \\
\hline & 5 & 3.4 & 13.28 & 12.31 & & 0.15 & 5.23 & 9.42 & 2.56 & & \\
\hline $25 \cdot 3,36-42$ & 48.00 & 3.55 & 13.75 & 5.92 & 7.54 & 0.20 & 6.70 & 8.44 & 2.94 & 0.73 & 0.389 \\
\hline Avg., $24-3$ to $25-3)^{d}$ & & 3.4 & & 5.92 & & & & & & & \\
\hline $26-4,5-10$ & 50 & 5.55 & 13.80 & 4.64 & 8.53 & 0.27 & 6.93 & 10.41 & 2.38 & 0.14 & \\
\hline $27-2,6-12$ & 45.50 & 4.00 & 14.01 & 8.03 & 6.73 & 0.25 & 6.07 & 11.03 & 2.31 & 0.17 & 0.435 \\
\hline $138-145^{a}$ & 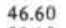 & 3. & 14 & 10. & & & & & & & \\
\hline $44-2,40-45^{a}$ & & 4.2 & & & & & 4.84 & & & & \\
\hline $46-1,45-50^{\mathrm{a}}$ & 49.10 & 4.32 & 13.20 & 13.77 & & 0.17 & 5.10 & 10.90 & 2.49 & 0.45 & 0.439 \\
\hline $\begin{array}{l}\text { (Avg., Holes 446A and } \\
446 \text { ) }\end{array}$ & 8.35 & 4.14 & 3.54 & 04 & 83 & 0.22 & 5.55 & 9.81 & 2.70 & 0.66 & 0.390 \\
\hline
\end{tabular}


TABLE 2 - Continued

\begin{tabular}{|c|c|c|c|c|c|c|c|c|c|c|c|c|c|c|c|c|c|}
\hline $\mathrm{Li}$ & $\mathrm{Rb}$ & Cs & Sr & B & $\mathrm{F}$ & v & $\mathrm{Cr}$ & $\mathrm{Cu}$ & $\begin{array}{c}\mathrm{Ni} \\
(\mathrm{ppm})\end{array}$ & Co & $\mathrm{Zn}$ & Th & U & TR & $\mathrm{La}$ & $\mathrm{Ce}$ & $\mathrm{Y}$ \\
\hline $\begin{array}{l}31 \\
26\end{array}$ & 9.4 & 0.8 & 180 & 10 & 330 & 290 & 260 & 72 & 52 & 40 & 82 & - & - & - & - & - & - \\
\hline $\begin{array}{l}26 \\
12\end{array}$ & $\begin{array}{l}8.2 \\
6.0\end{array}$ & $\begin{array}{r}0.6 \\
<0.5\end{array}$ & $\begin{array}{l}170 \\
170\end{array}$ & $\begin{array}{l}\sim 10 \\
<10\end{array}$ & $\begin{array}{l}390 \\
400\end{array}$ & $\begin{array}{l}270 \\
260\end{array}$ & $\begin{array}{l}230 \\
200\end{array}$ & $\begin{array}{l}70 \\
79\end{array}$ & $\begin{array}{r}44 \\
135\end{array}$ & $\begin{array}{l}43 \\
40\end{array}$ & $\begin{array}{r}72 \\
105\end{array}$ & - & - & - & - & - & - \\
\hline 5 & 3.1 & & 150 & $<10$ & $\begin{array}{l}450 \\
250\end{array}$ & 300 & 190 & $\begin{array}{l}79 \\
78\end{array}$ & $\begin{array}{r}135 \\
84\end{array}$ & $\begin{array}{l}40 \\
47\end{array}$ & $\begin{array}{r}105 \\
94\end{array}$ & $\bar{z}$ & $\overline{-}$ & $z$ & $\bar{z}$ & $\bar{z}$ & $=$ \\
\hline 10 & 7.9 & $<0.5$ & 150 & $<10$ & 240 & 290 & 190 & $\begin{array}{l}78 \\
78\end{array}$ & $\begin{array}{l}84 \\
56\end{array}$ & $\begin{array}{l}41 \\
38\end{array}$ & $\begin{array}{r}94 \\
105\end{array}$ & $\overline{-}$ & $\overline{-}$ & 30 & 2.28 & 7.53 & 10.71 \\
\hline 10 & 3.5 & $<0.5$ & 110 & $<10$ & 120 & 250 & 200 & 100 & 130 & 44 & 80 & $=$ & - & 20 & 1.58 & 4.78 & 5.90 \\
\hline 12 & 5.0 & $<0.5$ & 120 & 11 & 150 & 250 & 230 & 52 & 130 & 44 & 85 & $<0.3$ & 0.7 & - & - & - & - \\
\hline 10 & 6.1 & 505 & 130 & 18 & 130 & 290 & 230 & 120 & 100 & 50 & 100 & - & $\bar{z}$ & $\overline{-}$ & $\overline{-}$ & z & - \\
\hline 10 & $\begin{array}{l}1.8 \\
4.7\end{array}$ & $\begin{array}{l}<0.5 \\
<0.5\end{array}$ & $\begin{array}{l}150 \\
132\end{array}$ & & 260 & $\begin{array}{l}320 \\
280\end{array}$ & $\begin{array}{l}230 \\
216\end{array}$ & $\begin{array}{l}76 \\
85\end{array}$ & $\begin{array}{l}110 \\
105\end{array}$ & $\begin{array}{l}40 \\
43\end{array}$ & $\begin{array}{l}72 \\
88\end{array}$ & $<0.3$ & 0.7 & 25 & 1.83 & $6 . \overline{15}$ & $\overline{8.30}$ \\
\hline $\begin{array}{l}10 \\
33\end{array}$ & $\begin{array}{l}4.7 \\
5.8\end{array}$ & & 140 & $\begin{array}{r}12 \\
<10\end{array}$ & $\begin{array}{l}180 \\
250\end{array}$ & $\begin{array}{l}280 \\
240\end{array}$ & $\begin{array}{l}216 \\
190\end{array}$ & $\begin{array}{l}85 \\
77\end{array}$ & $\begin{array}{r}105 \\
84\end{array}$ & $\begin{array}{l}43 \\
38\end{array}$ & $\begin{array}{l}88 \\
75\end{array}$ & -0.3 & & & & - & $\begin{array}{l}8.30 \\
-\end{array}$ \\
\hline 13 & 4.2 & $<0.5$ & 150 & $<10$ & 260 & 240 & 220 & 70 & 100 & 48 & 92 & - & - & 40 & 2.80 & 9.32 & 12.80 \\
\hline 23 & 5.0 & $<0.5$ & 145 & $<10$ & 255 & 240 & 205 & 74 & 92 & 43 & 84 & - & - & 40 & 2.80 & 9.32 & 12.80 \\
\hline 32 & $\begin{array}{r}9.1 \\
14.0\end{array}$ & ${ }^{\circ} 0.8$ & $\begin{array}{l}150 \\
170\end{array}$ & $<10$ & $\begin{array}{l}130 \\
330\end{array}$ & $\begin{array}{l}330 \\
270\end{array}$ & $\begin{array}{r}290 \\
260\end{array}$ & 57 & 92 & 56 & $\begin{array}{r}75 \\
\end{array}$ & - & - & - & - & - & - \\
\hline 13 & 14.0 & 0.8 & $\begin{array}{l}170 \\
150\end{array}$ & $\begin{array}{r}22 \\
<10\end{array}$ & $\begin{array}{l}330 \\
270\end{array}$ & $\begin{array}{l}270 \\
210\end{array}$ & $\begin{array}{l}260 \\
150\end{array}$ & $\begin{array}{l}64 \\
97\end{array}$ & $\begin{array}{l}50 \\
65\end{array}$ & $\begin{array}{r}81 \\
150\end{array}$ & 100 & $\bar{z}$ & $\bar{z}$ & $\bar{z}$ & $\bar{z}$ & $z$ & $\bar{z}$ \\
\hline- & - & - & - & - & - & - & - & - & - & 100 & - & - & - & - & - & - & - \\
\hline 10 & 13.0 & $<0.5$ & 160 & 15 & 270 & 240 & 150 & 52 & 45 & 160 & 100 & - & - & - & - & - & - \\
\hline$\overline{-}$ & - & - & 220 & $<10$ & 300 & 400 & 120 & 95 & 80 & 45 & 10 & - & - & - & - & - & $\bar{z}$ \\
\hline$\overline{-}$ & I & $\overline{-}$ & $=$ & $\overline{-}$ & $\bar{z}$ & $\overline{-}$ & $=$ & $\bar{z}$ & $\overline{-}$ & $=$ & $=$ & = & $\bar{z}$ & $\overline{-}$ & - & - & - \\
\hline 18 & 13 & $<0.5$ & 190 & $<10$ & 270 & 290 & 50 & 74 & 34 & $\overline{53}$ & $\overline{110}$ & -50.3 & $<\overline{0.2}$ & - & - & $\bar{z}$ & $\overline{-}$ \\
\hline 10 & 16 & 1.6 & 180 & 12 & 270 & 250 & 65 & 75 & 36 & 54 & 105 & - & 80.2 & 70 & $\overline{7.98}$ & $14 . \overline{56}$ & 26.74 \\
\hline $\bar{z}$ & $\bar{z}$ & $z$ & $\bar{z}$ & $\overline{-}$ & $\bar{z}$ & $\bar{z}$ & $\overline{-}$ & $\bar{z}$ & $\overline{-}$ & $\bar{z}$ & $\bar{z}$ & $\overline{-}$ & $\overline{-}$ & $\overline{-}$ & $\bar{z}$ & $\bar{z}$ & $\overline{-}$ \\
\hline - & - & - & - & - & - & - & - & - & - & - & - & - & - & - & - & $\bar{z}$ & $z$ \\
\hline - & - & - & - & - & - & - & - & - & - & - & - & - & - & - & - & - & - \\
\hline - & - & - & - & - & - & - & - & - & - & - & & - & - & - & - & - & - \\
\hline - & - & - & - & - & - & - & - & - & - & - & - & - & - & - & - & - & - \\
\hline 16 & 9.2 & 0.6 & 183 & 11 & 255 & 277 & 207 & 74 & 73 & 67 & 90 & 0.3 & 0.7 & 40 & 3.66 & 9.05 & 14.04 \\
\hline 7.3 & 6.0 & $<0.5$ & 140 & $\begin{array}{l}<10 \\
<10\end{array}$ & 160 & 340 & 270 & 75 & 87 & 46 & 125 & $=$ & $=$ & - & - & - & - \\
\hline $\begin{array}{l}6.9 \\
7.5\end{array}$ & $\begin{array}{l}7.6 \\
3.4\end{array}$ & $\begin{array}{r}1.4 \\
<0.5\end{array}$ & $\begin{array}{l}160 \\
120\end{array}$ & $<10$ & $\begin{array}{l}150 \\
170\end{array}$ & $\begin{array}{l}340 \\
440\end{array}$ & $\begin{array}{l}280 \\
250\end{array}$ & $\begin{array}{l}71 \\
72\end{array}$ & 110 & 54 & 120 & $<0.3$ & $<0.1$ & $\overline{50}$ & $\overline{390}$ & $\overline{9}-\bar{s}$ & - \\
\hline & & & & $\bar{z}$ & & & & & 97 & 48 & 100 & $=$ & $\bar{z}$ & 50 & 3.90 & 9.50 & 11.10 \\
\hline $\begin{array}{c}8.8 \\
16\end{array}$ & 7.9 & $<0.5$ & 120 & - & 230 & 400 & 210 & 58 & 88 & 81 & 130 & & - & - & - & - & - \\
\hline $\begin{array}{l}16 \\
12\end{array}$ & 17.5 & 0.7 & 130 & $<10$ & 350 & 470 & 190 & 35 & 43 & 30 & 95 & $<0.3$ & $<0.1$ & - & - & - & - \\
\hline $\begin{array}{l}12 \\
18\end{array}$ & $\begin{array}{l}9.8 \\
5.1\end{array}$ & $\begin{array}{r}0.5 \\
<0.5\end{array}$ & $\begin{array}{l}130 \\
150\end{array}$ & $\begin{array}{l}<10 \\
<10\end{array}$ & $\begin{array}{l}460 \\
520\end{array}$ & $\begin{array}{l}600 \\
370\end{array}$ & 200 & 56 & 62 & 74 & 150 & $=$ & $=$ & 100 & 72 & & 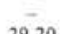 \\
\hline & 10.8 & $<0.5$ & 140 & $<10$ & $\begin{array}{l}520 \\
443\end{array}$ & $\begin{array}{l}370 \\
480\end{array}$ & $\begin{array}{l}2100 \\
200\end{array}$ & $\begin{array}{l}56 \\
49\end{array}$ & $\begin{array}{l}80 \\
62\end{array}$ & $\begin{array}{l}44 \\
49\end{array}$ & $\begin{array}{l}100 \\
115\end{array}$ & $<\overline{0.3}$ & $<\overline{0.1}$ & $\begin{array}{l}100 \\
100\end{array}$ & $\begin{array}{l}7.2 \\
7.2\end{array}$ & $\begin{array}{l}17.3 \\
17.3\end{array}$ & $\begin{array}{l}29.20 \\
29.20\end{array}$ \\
\hline 7.4 & 3.2 & & 110 & 23 & 120 & 250 & 270 & 66 & 160 & 47 & 72 & & - & - & & & \\
\hline 9.2 & 2.0 & $<0.5$ & 140 & $<10$ & 290 & 270 & 290 & 72 & 92 & 42 & 86 & $<0.3$ & 0.2 & 60 & 9.5 & 13.1 & 13.56 \\
\hline 10 & 7.8 & $<0.5$ & 140 & $<10$ & 140 & 265 & 300 & 68 & 100 & 45 & 90 & 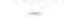 & & & & & \\
\hline 12 & 5.0 & $<0.5$ & 130 & $<10$ & 100 & 265 & 280 & 46 & 98 & 43 & 85 & - & - & 30 & 1.9 & 7.3 & 8.8 \\
\hline 11 & 6.4 & $<0.5$ & 135 & $<10$ & 120 & 265 & 290 & 56 & 99 & 44 & 87 & $=$ &.- & 30 & 1.9 & 7.3 & 8.8 \\
\hline 10.5 & 6.8 & 0.6 & 171 & $<10$ & 244 & 364 & 271 & 61 & 92 & so & 112 & $<0.3$ & 0.1 & 60 & 5.6 & 11.8 & 15.7 \\
\hline 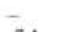 & - & - & 420 & $<10$ & 250 & 195 & 190 & 58 & 120 & 30 & - & - & - & - & - & - & - \\
\hline 7.1 & 23 & 1.2 & & $<10$ & 310 & 320 & & 56 & 32 & 30 & 76 & 2.7 & 0.8 & - & - & - & - \\
\hline 8.5 & 16 & $<0.5$ & 230 & $<10$ & 120 & 240 & 2 & 42 & 170 & 42 & 70 & 1.4 & 0.8 & - & - & - & - \\
\hline $\begin{array}{l}7.8 \\
60\end{array}$ & $\begin{array}{l}19 \\
30\end{array}$ & 0.8 & 340 & $<10$ & 227 & 252 & 1 & 52 & 100 & 34 & 73 & 2.0 & 0.8 & - & - & - & - \\
\hline $\begin{array}{l}6.0 \\
5.1\end{array}$ & 3.0 & & 120 & $<10$ & 200 & 225 & 30 & 68 & 61 & 44 & 71 & - & - & - & - & - & - \\
\hline 5.1 & 10 & $<0.5$ & $\begin{array}{l}150 \\
250\end{array}$ & $<10$ & 220 & $\begin{array}{l}250 \\
175\end{array}$ & 24 & 67 & 58 & 43 & 100 & - & - & - & - & - & - \\
\hline 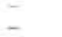 & - & - & $\begin{array}{l}250 \\
2500\end{array}$ & $\begin{array}{l}<10 \\
<10\end{array}$ & $\begin{array}{l}150 \\
220\end{array}$ & $\begin{array}{l}175 \\
175\end{array}$ & 3. & $\begin{array}{l}54 \\
65\end{array}$ & $\begin{array}{l}80 \\
90\end{array}$ & $\begin{array}{l}40 \\
35\end{array}$ & - & - & - & - & - & - & - \\
\hline - & - & 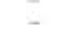 & 170 & $<10$ & 230 & 200 & $\begin{array}{l}23 \\
24\end{array}$ & $\begin{array}{l}63 \\
70\end{array}$ & $\begin{array}{r}90 \\
100\end{array}$ & $\begin{array}{l}35 \\
45\end{array}$ & $=$ & $z$ & $\bar{z}$ & $\bar{z}$ & $\overline{-}$ & - & = \\
\hline & & - & 145 & $<10$ & 170 & 290 & 310 & 63 & 110 & 46 & - & $=$ & $=$ & $=$ & 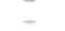 & 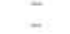 & $\overline{-}$ \\
\hline 6.6 & 7.6 & 0.8 & 160 & $<10$ & 180 & 280 & 300 & 66 & 110 & 53 & 89 & - & - & - & - & - & - \\
\hline 5.8 & 9.0 & 0.6 & 183 & $<10$ & 195 & 228 & 275 & 64 & 91 & 44 & 95 & - & - & - & - & - & - \\
\hline 6.2 & 16 & 0.6 & 310 & $<10$ & 370 & 220 & 150 & 140 & 78 & 44 & 170 & - & - & - & - & - & - \\
\hline 6 & 9.3 & 1.0 & & $<10$ & 33 & & 80 & & 51 & 47 & 170 & - & - & & - & - & - \\
\hline 6.5 & 8.4 & $<0.5$ & 3. & $<10$ & 42 & 17 & 70 & 16 & 53 & 48 & 180 & - & - & - & - & - & - \\
\hline 6.0 & 33 & 2.2 & & $<10$ & 58 & 24 & 130 & ? & 55 & 43 & 120 & & & - & & - & - \\
\hline & & & 41 & $<1$ & 40 & 2 & 9 & $2 !$ & 80 & 40 & & & - & - & - & - & - \\
\hline $\begin{array}{l}8.8 \\
6.2\end{array}$ & 5.6 & & 3. & $<10$ & 49 & 31 & 140 & 13 & 6 & 46 & 14 & $\therefore$ & $=$ & - & - & - & - \\
\hline $\begin{array}{l}6.2 \\
6.4\end{array}$ & 14 & 1,2 & & $<10$ & 62 & & & 10 & 6 & 48 & & 1.9 & 0.3 & & - & & - \\
\hline & 8.6 & $<0.5$ & $\begin{array}{l}340 \\
360\end{array}$ & $<10$ & $\begin{array}{r}500 \\
540\end{array}$ & $\begin{array}{l}290 \\
350\end{array}$ & $\begin{array}{r}160 \\
30\end{array}$ & 17 & 98 & 53 & $\begin{array}{l}120 \\
170\end{array}$ & - & - & - & I & z & - \\
\hline 7.5 & 11.3 & 1.5 & 350 & $<10$ & $\begin{array}{l}54 \\
52\end{array}$ & 32 & 95 & 12 & & 49 & 145 & - & - & - & - & $=$ & $\overline{-}$ \\
\hline 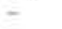 & & & & $<10$ & 40 & 20 & 50 & 16 & 4 & 34 & - & 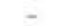 & - & 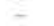 & 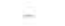 & - & - \\
\hline & 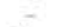 & - & 47 & $<10$ & 42 & 26 & 30 & 15 & 40 & 38 & 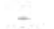 & . & - & 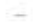 & 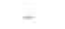 & & - \\
\hline 11 & 20 & 0.8 & & $<10$ & 60 & 37 & 30 & 8 & 3 & 45 & 18 & - & - & 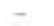 & 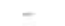 & - & 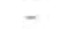 \\
\hline 1 & 20 & 0. & & $<10$ & 47 & 27 & 37 & 13 & 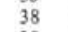 & 39 & & - & - & & & & 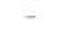 \\
\hline 7.4 & 20 & $<0.5$ & & $<10$ & 47 & 41 & 40 & 10 & 3 & 48 & i) & 1.7 & 0.7 & - & - & & - \\
\hline 7.0 & 13 & $<0.5$ & & $<10$ & 66 & 38 & 20 & & 2 & 5. & 18 & . & - & 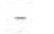 & 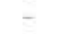 & - & 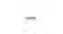 \\
\hline 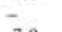 & & & & $<$ & 43 & & 3 & & 3 & 3 & & - & - & - & - & - & - \\
\hline 7.6 & 13 & $<0.5$ & & & 5 & & & & 2 & & & - & - & - & - & & - \\
\hline 9. & 15 & 2.4 & & $<$ & 4 & 23 & 180 & & 9 & 40 & 130 & - & - & - & - & - & - \\
\hline - & - & - & & $<$ & 45 & 27 & 54 & & 55 & 7 & - & - & - & & - & & - \\
\hline 7 & 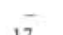 & 3 & & $<1$ & 50 & 27 & 6. & & 60 & 37 & 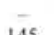 & $\therefore$ & $\overline{0.5}$ & - & - & - & - \\
\hline 7.5 & 17 & 1.3 & 35 & $<10$ & 41 & 36 & 5 & 12 & 4 & 45 & 14 & 1 & 0.5 & & - & & - \\
\hline $\begin{array}{l}7.5 \\
80\end{array}$ & 178 & 1.3 & 41 & $<10$ & 46 & 30 & 58 & 16 & 5. & 41 & 14 & 1.9 & 0.5 & - & - & - & - \\
\hline $\begin{array}{l}8.0 \\
5.9\end{array}$ & $\begin{array}{c}8.9 \\
17\end{array}$ & $\begin{array}{l}1.9 \\
1.8\end{array}$ & $\begin{array}{l}400 \\
440\end{array}$ & $\begin{array}{l}<10 \\
<10\end{array}$ & $\begin{array}{l}420 \\
540\end{array}$ & $\begin{array}{l}285 \\
240\end{array}$ & $\begin{array}{r}130 \\
40\end{array}$ & $\begin{array}{l}150 \\
120\end{array}$ & $\begin{array}{l}63 \\
40\end{array}$ & $\begin{array}{l}44 \\
46\end{array}$ & $\begin{array}{l}130 \\
160\end{array}$ & $\overline{-}$ & $z$ & $\bar{z}$ & $\bar{z}$ & 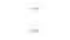 & 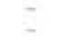 \\
\hline- & - & - & 38 & $<10$ & 440 & 37 & 8 & 270 & 75 & $4 i$ & - & - & - & & & - & - \\
\hline & 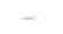 & - & 450 & $<10$ & 470 & 210 & 55 & 240 & 50 & 50 & 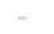 & 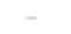 & 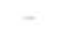 & 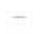 & & - & - \\
\hline - & - & - & - & - & - & - & - & - & - & 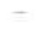 & - & - & - & 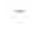 & 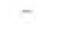 & - & - \\
\hline 7.52 & 14.9 & 1.3 & 490 & $<10$ & 488 & 272 & 66 & 119 & 56 & 46 & 151 & 1.9 & $3-7$ & - & - & - & - \\
\hline
\end{tabular}


TABLE 2 - Continued

\begin{tabular}{|c|c|c|c|c|c|c|c|c|c|c|c|}
\hline Sample (interval in $\mathrm{cm}$ ) & Q & Or & $\mathrm{Ab}$ & An & $\mathrm{Ne}$ & $\begin{array}{l}\mathrm{Di} \\
(\%)\end{array}$ & $\mathrm{Hy}$ & OI & Mt & II & Ap \\
\hline $442 \mathrm{~B}-3-3,140-146$ & 3.62 & 2.00 & 23.51 & 30.75 & - & 21.53 & 7.64 & - & 6.23 & 2.45 & 0.28 \\
\hline $4-2,35-40$ & 1.07 & 2.05 & 26.47 & 30.55 & - & 24.28 & 5.06 & - & 6.43 & 2.39 & 0.29 \\
\hline $5-2,65-70$ & 0.30 & 1.21 & 26.96 & 30.68 & - & 18.12 & 14.08 & - & 6.02 & 2.38 & 0.24 \\
\hline $6 \cdot 3,27-35$ & 0.04 & 1.87 & 22.17 & 29.05 & - & 18.36 & 21.32 & - & 3.59 & 3.50 & 0.09 \\
\hline $7-1,35-42$ & - & - & - & - & - & - & - & - & - & - & - \\
\hline $8-1,80-85$ & - & - & - & - & - & - & - & - & - & - & - \\
\hline $8-3,120-125$ & - & - & - & - & - & - & - & - & - & - & - \\
\hline $8-4,60-65$ & - & - & - & - & - & - & - & - & - & - & - \\
\hline $8-6,50-55$ & - & - & - & - & - & - & - & - & - & - & - \\
\hline (Avg., $7-1$ to $8-6$ ) & - & 0.99 & 25.16 & 31.40 & - & 14.30 & 16.34 & 1.76 & 6.34 & 2.33 & 0.26 \\
\hline $8-6,135-140$ & - & - & - & - & - & - & - & - & - & - & - \\
\hline $8-7,47-52$ & - & - & - & - & - & - & - & - & - & - & - \\
\hline (Avg., 8-6 and 8-7) & 1.27 & 1.56 & 28.63 & 28.13 & - & 14.97 & 14.09 & - & 8.06 & 2.41 & 0.11 \\
\hline $9-1,10-15$ & 3.40 & 1.65 & 23.67 & 32.97 & - & 14.68 & 13.84 & - & 5.83 & 2.02 & 0.26 \\
\hline $10-1,78-84$ & 3.90 & 2.34 & 26.12 & 29.60 & - & 18.54 & 5.69 & - & 10.31 & 3.09 & 0.40 \\
\hline $10-1,93-96^{a}$ & - & 2.09 & 21.01 & 23.38 & 3.46 & 36.04 & - & 10.55 & - & 3.17 & 0.29 \\
\hline $\begin{array}{l}\text { (Avg., uppet } 59 \mathrm{~m} \text {. of } \\
\text { the hole) }\end{array}$ & - & - & - & - & - & - & - & - & - & - & - \\
\hline $10-1,118-121^{b}$ & 1.67 & 2.41 & 27.59 & 26.58 & - & 25.58 & 2.43 & - & 9.30 & 3.16 & 0.34 \\
\hline $14-1,35-40^{a, b}$ & - & 1.30 & 24.56 & 31.19 & - & 23.05 & 5.09 & 11.51 & - & 3.21 & - \\
\hline $14-1,85-90 \mathrm{a}, \mathrm{b}$ & - & 1.54 & 25.49 & 30.06 & - & 23.34 & 4.55 & 11.97 & - & 2.99 & - \\
\hline $14-1,115-120^{\mathrm{a}, \mathrm{b}}$ & - & 1.42 & 25.47 & 30.40 & - & 23.06 & 4.56 & 12.12 & - & 2.89 & - \\
\hline $16-1,35-47$ & 2.31 & 2.40 & 24.62 & 32.24 & - & 21.50 & 6.33 & - & 7.69 & 2.56 & 0.36 \\
\hline $17-1,50-55$ & 2.24 & 2.39 & 27.34 & 28.68 & - & 23.10 & 4.25 & - & 8.17 & 3.34 & 0.53 \\
\hline $17-1,84-86^{\mathrm{a}, \mathrm{b}}$ & - & 1.50 & 25.48 & 28.74 & - & 24.11 & 8.44 & 8.53 & - & 3.13 & - \\
\hline $17-1,117-120^{\mathrm{a}, \mathrm{b}}$ & - & 1.50 & 25.25 & 28.67 & - & 23.54 & 10.13 & 7.68 & - & 3.15 & - \\
\hline $17-2,10-15 \mathrm{a}, \mathrm{b}$ & - & 1.49 & 22.37 & 29.72 & - & 24.31 & 13.56 & 5.44 & - & 3.06 & - \\
\hline $19-2,65-70^{a, b}$ & - & 1.48 & 21.65 & 31.19 & - & 21.93 & 18.20 & 2.30 & - & 3,18 & - \\
\hline $20-1,57-61^{1}, \mathrm{~b}$ & 0.47 & 1.54 & 20.36 & 31.56 & - & 21.98 & 20.88 & - & - & 3.16 & - \\
\hline $\begin{array}{l}\text { (Avg., lower } 110 \mathrm{~m} \text { of } \\
\text { the hole) }\end{array}$ & - & - & - & - & - & - & - & - & - & - & - \\
\hline (Avg., Hole 442B) & - & - & - & - & - & - & - & - & - & - & - \\
\hline $443-53-1,15-20$ & - & 0.66 & 23.95 & 33.05 & - & 17.27 & 11.67 & 6.01 & 4.28 & 2.85 & 0.28 \\
\hline $54-4,115-120$ & - & 0.84 & 23.74 & 31.78 & - & 18.17 & 10.91 & 7.41 & 4.23 & 2.66 & 0.26 \\
\hline $54-7,63-67$ & - & 0.42 & 22.79 & 30.99 & - & 20.87 & 11.14 & 6.29 & 4.14 & 2.82 & 0.25 \\
\hline $57-2,105-108^{\mathrm{a}, \mathrm{b}}$ & - & 1.66 & 21.06 & 31.98 & - & 22.02 & 10.06 & 9.78 & - & 3.37 & - \\
\hline $57-3,0-5$ & - & 1.41 & 26.73 & 30.64 & - & 15.84 & 15.87 & 0.48 & 6.16 & 2.45 & 0.45 \\
\hline $58-2,71-75$ & - & - & - & - & - & - & - & - & - & - & - \\
\hline $58-2,125-128$ & - & - & - & - & - & - & - & - & - & - & - \\
\hline $58-3,121-125$ & - & - & - & - & - & - & - & - & - & - & - \\
\hline (Avg., 58-2 to 58-3) & 1.30 & 2.41 & 27.31 & 28.22 & - & 16.29 & 12.81 & - & 7.67 & 3.48 & 0.42 \\
\hline $61-1,106-110$ & - & 0.55 & 22.76 & 33.05 & - & 16.43 & 16.23 & 1.95 & 6.52 & 2.29 & 0.23 \\
\hline $62-1,88-93$ & 0.70 & 0.66 & 23.95 & 32.15 & - & 22.11 & 13.69 & - & 4.55 & 2.56 & 0.27 \\
\hline $63-8,89-94$ & - & - & - & - & - & - & - & - & - & - & - \\
\hline $64-2,84-86$ & - & - & - & - & - & - & - & - & - & - & - \\
\hline (Avg., 63-8 and 64-2) & 1.06 & 0.67 & 24.80 & 32.04 & - & 18.37 & 12.36 & - & 6.22 & 2.52 & 0.25 \\
\hline (Avg., Hole 443) & - & - & - & - & - & - & - & - & - & - & - \\
\hline $444 \mathrm{~A}-20-1,39-43^{a}$ & - & - & - & - & - & - & - & - & - & - & - \\
\hline $20-1,77-81^{\mathrm{c}}$ & - & - & - & - & - & - & - & - & - & - & - \\
\hline $20-4,41-45 \mathrm{c}$ & - & 14.22 & 22.09 & 20.59 & 5.99 & 18.88 & - & 10.43 & 5.43 & 3.82 & 0.35 \\
\hline (Avg., 20-1 to $20-4)^{d}$ & 0.57 & 1.19 & 23.81 & 33.91 & - & 19.43 & 11.76 & - & 6.04 & 2.94 & 0.35 \\
\hline $25-3,111-115$ & - & - & - & - & - & - & - & - & - & - & - \\
\hline $26-1,76-80$ & - & - & - & - & - & - & - & - & - & - & - \\
\hline $26-3,75-79 a$ & - & - & - & - & - & - & - & - & - & - & - \\
\hline $27 \cdot 1,131-135 \mathrm{a}$ & - & $-\cdot$ & - & - & - & - & - & - & - & - & - \\
\hline $27-3,145-150^{\mathrm{a}}$ & - & - & - & - & - & - & - & - & - & - & - \\
\hline $27-4,119-123^{a}$ & - & - & - & - & - & - & - & - & - & - & - \\
\hline $27-5,61-65$ & - & - & - & - & - & - & - & - & - & - & - \\
\hline$(\text { Avg., 26-1 to } 27-5)^{d}$ & - & 1.74 & 23.39 & 30.89 & - & 22.05 & 7.12 & 9.18 & 5.45 & 2.75 & 0.37 \\
\hline $446 \mathrm{~A}-5-1,4-10$ & 5.61 & 4.18 & 21.12 & 22.71 & - & 19.05 & 12.78 & - & 6.51 & 7.30 & 0.77 \\
\hline $8-2,144-150$ & 5.79 & 1.81 & 21.01 & 25.60 & - & 14.25 & 14.61 & - & 8.27 & 7.96 & 0.72 \\
\hline $9-3,90-95$ & 7.12 & 2.41 & 22.52 & 23.42 & - & 14.98 & 10.87 & - & 9.61 & 8.13 & 0.94 \\
\hline $10-5,97-102$ & - & 9.44 & 18.92 & 20.49 & 3.47 & 25.16 & - & 2.70 & 7.67 & 10.37 & 1.81 \\
\hline $14-3,97-102^{a}$ & 1.65 & 1.90 & 20.75 & 24.06 & - & 20.51 & 23.48 & - & - & 7.00 & 0.65 \\
\hline $15-4,38-43$ & 9.51 & 2.72 & 22.55 & 24.47 & - & 15.52 & 8.40 & - & 9.70 & 7.37 & 0.77 \\
\hline $17, \mathrm{CC}, 24-32$ & 3.74 & 7.58 & 23.16 & 26.15 & - & 13.59 & 6.04 & - & 9.08 & 8.51 & 1.49 \\
\hline $19-3,0-8$ & - & - & - & - & - & - & - & - & - & - & - \\
\hline $19-3,67-73$ & - & - & - & - & - & - & - & - & - & - & - \\
\hline (Avg., 19-3) & 4.83 & 2.22 & 25.24 & 24.06 & - & 15.94 & 11.14 & - & 7.84 & 7.82 & 0.91 \\
\hline $21-2,82-88^{a}$ & - & - & - & - & - & - & - & - & - & - & - \\
\hline $21-5,140-146^{\mathrm{a}}$ & - & - & - & - & - & - & - & - & - & - & - \\
\hline $21-6,53-58$ & - & - & - & - & - & - & - & - & - & - & - \\
\hline (Avg., 21-2 to $21-6)^{d}$ & 5.12 & 3.18 & 22.59 & 21.22 & - & 19.07 & 17.30 & - & 2.92 & 7.66 & 0.92 \\
\hline $21-6,69-74$ & 2.66 & 4.43 & 26.14 & 24.56 & - & 14.14 & 11.28 & - & 8.04 & 7.89 & 0.88 \\
\hline $22-1,132-137$ & - & - & - & - & - & - & - & - & - & - & - \\
\hline $22-4,0-6^{\mathrm{a}}$ & - & - & - & - & - & - & - & - & - & - & - \\
\hline (Avg., 22-1 and 22-4) & 2.75 & 3.76 & 24.57 & 22.15 & - & 12.91 & 14.06 & - & 4.11 & 9.28 & 1.04 \\
\hline $23 \cdot 6,78-83$ & 5.50 & 2.53 & 22.33 & 26.38 & - & 15.37 & 10.87 & - & 9.20 & 7.02 & 0.82 \\
\hline $24-3,78-85^{a}$ & - & - & - & - & - & - & - & - & - & - & - \\
\hline $25-2,46-51^{a}$ & - & - & - & - & - & - & - & - & - & $=$ & - \\
\hline $25-3,36-42$ & - & - & - & - & - & - & - & - & - & - & - \\
\hline$(\text { Avg., 24-3 to } 25-3)^{d}$ & 3.75 & 4.22 & 23.40 & 21.78 & - & 20.14 & 16.19 & - & 2.92 & 6.70 & 0.88 \\
\hline $26-4,5-10$ & 3.50 & 0.84 & 20.53 & 26.98 & - & 18.62 & 15.12 & - & 6.83 & 6.87 & 0.82 \\
\hline $27-2,6-12$ & 5.34 & 1.02 & 19.83 & 28.00 & - & 19.39 & 6.35 & - & 11.05 & 7.77 & 1.05 \\
\hline $446-41, \mathrm{CC}, 138-145^{\mathrm{a}}$ & - & 13.36 & 5.48 & 18.55 & 12.04 & 37.81 & - & 4.61 & - & 7.51 & 0.65 \\
\hline $44-2,40-45$ & 0.37 & 5.37 & 23.59 & 21.32 & - & 23.33 & 16.94 & - & - & 8.12 & 0.92 \\
\hline $46-1.45-50^{3}$ & 0.75 & 2.66 & 21.07 & 23.53 & - & 23.20 & 19.50 & - & - & 8.20 & 1.04 \\
\hline $\begin{array}{l}\text { (Avg., Holes 446A and } \\
446 \text { ) }\end{array}$ & - & - & - & - & - & - & - & - & - & - & - \\
\hline
\end{tabular}

${ }^{2} \mathrm{X}$-Ray-fluorescence method.

Glass.

Alkaline-basilt sill.

Analysts: Vta for iron, Andreeva, S. M. Chernogorova, V. I. Pavlutzkaja, Institute of Geochemistry, Academy of Sciences of the U.S.S.R., Moscow. 


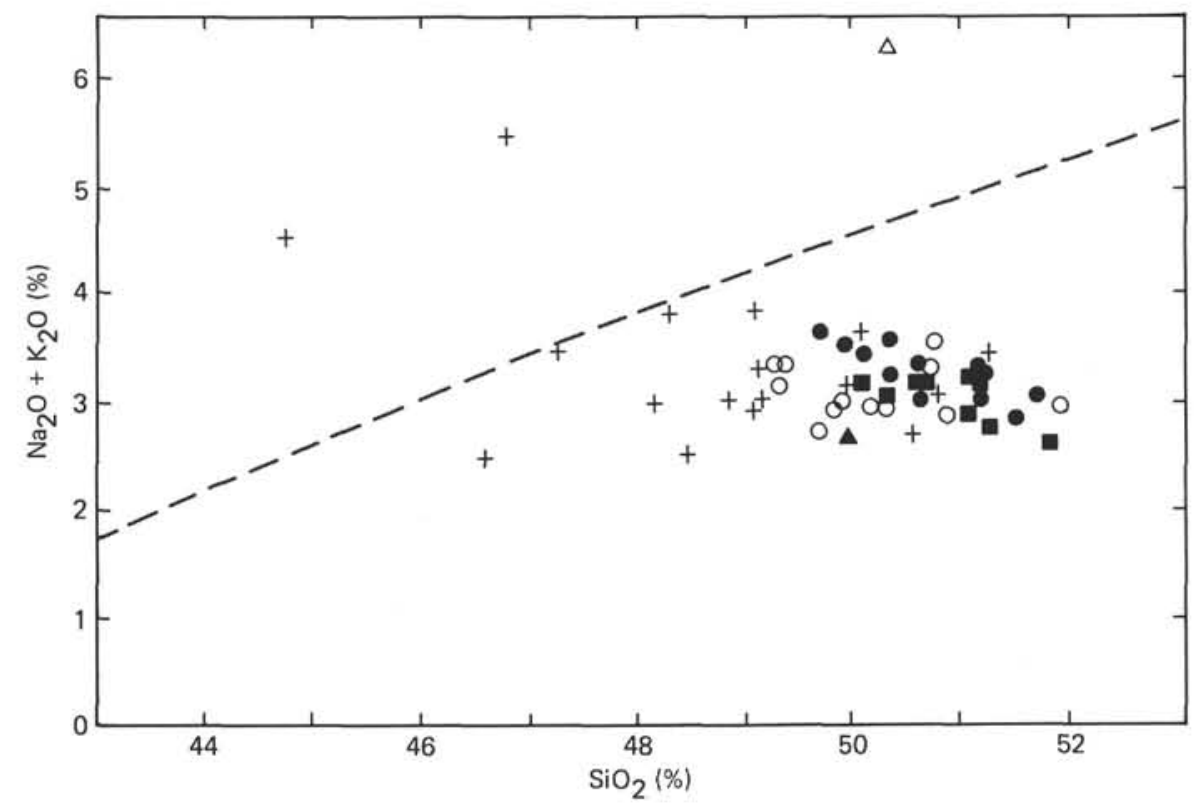

Figure 3. Alkali-silica diagram. Dashed line is the statistical boundary of tholeiitic basalts described by Irvine and Baragar (1971). Symbols are explained in Figure 1.

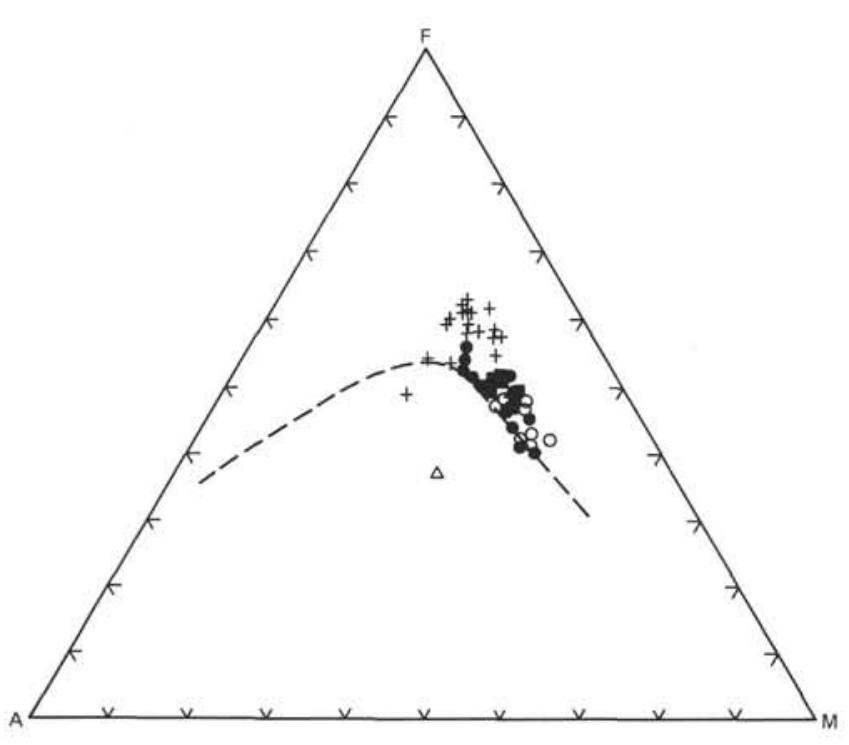

Figure 4. Positions of basalts in an FMA diagram. Dashed line is the statistical boundary of tholeiitic basalts described by Irvine and Baragar (1971). Symbols are explained in Figure 1.

originated beyond rift zones, and this is characteristic not only of sills (Hart, 1976), but also of lava flows and pillow basalts (Table 2).

The differences in composition of basalts in the northern Philippine Sea are caused both by differences in composition of parent magma and by differentiation. This is shown by the olivine-plagioclase-diopside diagram (Figure 5) and by variation diagrams (Figure 7).

To compare the results of analyses (partly using $\mathrm{X}$-ray fluorescence) presented on the three-component diagram of olivine-plagioclase-diopside, recalculations for normative composition were performed for ferrous oxide, which is probably reliable, because Mössbauer spectroscopy of glass detected not more than 15 per cent ferric iron. Also, hypersthene is recalculated for olivine and quartz $(3 \mathrm{Ol}+1 \mathrm{Q}=4 \mathrm{OPx})$, as is albite for nepheline and quartz $(3 \mathrm{Ne}+2 \mathrm{Q}=5 \mathrm{Ab})$.

Figure 5 shows that practically all the basalts were formed with plagioclase and olivine removal from the melt. This phenomenon is noticed in thin sections, where phenocrysts of these minerals, particularly plagioclase, occur in glass. A tendency toward removal of clinopyroxene from the melt is seen for Holes 442B and 446A. However, clinopyroxene phenocrysts in a slightly devitrified groundmass occur only in thin sections of Hole 446A alkaline basalts (Sample 446A-13-1, $17 \mathrm{~cm}$ ). In general, Figure 5 indicates little differentiation of Hole 443 basalts and substantial differentiation of basalts of Holes 442B and 446A.

This feature is most conspicuous in the variation diagrams: in Figure 7, Hole 442B and Hole 446A basalts reach their maximum and have the highest range of differentiation indexes (Hole 442B), with strong enrichment of the final melts in iron, titanium, and alkalis (particularly Hole 446A), and simultaneous decrease in magnesium content. The limited evolution of the Hole 443 basalts suggests inconsiderable differentiation. This also applies to glass of Hole $442 \mathrm{~B}$ basalts. Figure 7 shows that basalts of each hole have independent trends. For Holes 442B and 443, these are either close to each other or subparallel, but quite distinctive with respect to such characteristic components as silica, iron, magnesium, nickel, and chromium. This probably indicates similar, but somewhat different, initial compositions of the parent magmas from which the two rock 


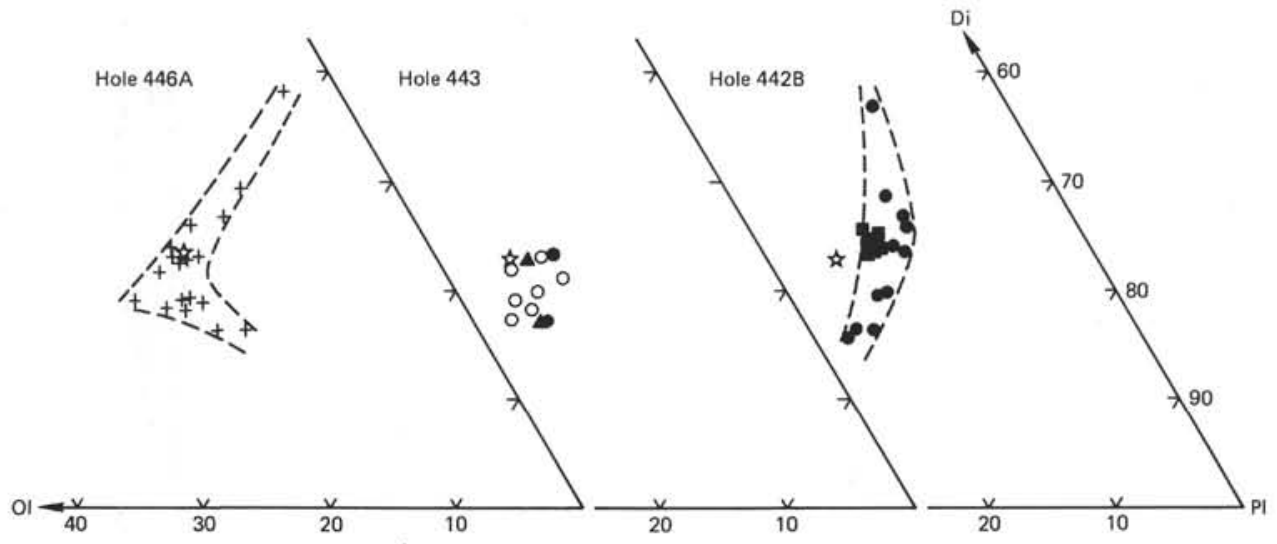

Figure 5. Basalts of three holes plotted on a normative Ol-Pl-Di diagram. Mean Ocean Ridge Basalt is indicated by stars (Dmitriev et al., 1976); other symbols are explained in Figure 1.

TABLE 3

Average Chemical Composition of Some Basalts

\begin{tabular}{|c|c|c|c|c|c|c|c|c|}
\hline \multicolumn{9}{|c|}{ Basalts of Leg 58 (from Table 2) } \\
\hline & \multicolumn{3}{|c|}{ Hole $442 B$} & \multirow{3}{*}{$\begin{array}{c}\text { Average, } \\
\text { Hole } 443 \\
\text { (12 samples) }\end{array}$} & \multirow{3}{*}{$\begin{array}{c}\text { Average, } \\
\text { Holes } 446 \mathrm{~A} \\
\text { and } 446 \\
\text { (24 samples) }\end{array}$} & \multirow{3}{*}{$\begin{array}{l}\text { TOPS } \\
\text { (Hart, } \\
1976 \text { ) }\end{array}$} & \multirow{2}{*}{\multicolumn{2}{|c|}{ MORB }} \\
\hline & Upper & Lower & $\overline{\text { A verage, }}$ & & & & & \\
\hline & $\begin{array}{c}59 \text { meters } \\
\text { (14 samples) }\end{array}$ & $\begin{array}{l}102 \text { meters } \\
\text { (11 samples) }\end{array}$ & $\begin{array}{l}\text { Hole } 442 \mathrm{~B} \\
\text { (25 samples) }\end{array}$ & & & & $\begin{array}{l}\text { (Hart, } \\
1976)\end{array}$ & $\begin{array}{c}\text { (Dmitriev et al., } \\
1976)\end{array}$ \\
\hline $\mathrm{SiO}_{2}(\%)$ & 49.16 & 50.76 & 49.72 & 48.72 & 48.35 & 49.09 & 49.92 & 49.96 \\
\hline $\mathrm{TiO}_{2}$ & 1.35 & 1.60 & 1.49 & 1.39 & 4.14 & 1.55 & 1.46 & 1.48 \\
\hline $\mathrm{Al}_{2} \mathrm{O}_{3}$ & 15.70 & 15.90 & 15.81 & 16.11 & 13.54 & 15.48 & 16.08 & 15.74 \\
\hline $\mathrm{Fe}_{2} \mathrm{O}_{3}$ & 5.16 & 9.70 & 9.39 & 4.31 & 6.04 & 943 & 9.26 & 2.48 \\
\hline $\mathrm{FeO}$ & 4.35 & 9.10 & 9.39 & 5.18 & 7.83 & 9.43 & 9.20 & 8.06 \\
\hline $\mathrm{MnO}$ & 0.12 & - & - & 0.14 & 0.22 & 0.18 & 0.17 & 0.18 \\
\hline $\mathrm{MgO}$ & 7.14 & 6.49 & 6.78 & 8.03 & 5.55 & 6.72 & 7.75 & 7.97 \\
\hline $\mathrm{CaO}$ & 10.90 & 11.74 & 11.36 & 10.85 & 9.81 & 10.66 & 11.21 & 11.36 \\
\hline $\mathrm{Na}_{2} \mathrm{O}$ & 2.94 & 2.87 & 2.91 & 2.83 & 2.70 & 2.73 & 2.79 & 2.63 \\
\hline $\mathrm{K}_{2} \mathrm{O}$ & 0.29 & 0.29 & 0.29 & 0.15 & 0.66 & 0.49 & 0.17 & 0.23 \\
\hline $\mathrm{P}_{2} \mathrm{O}_{5}$ & 0.103 & - & - & 0.12 & 0.39 & 0.18 & 0.15 & - \\
\hline $\mathrm{H}_{2} \mathrm{O}+$ & 1.96 & - & - & 1.08 & 1.24 & 2.65 & 0.77 & - \\
\hline $\mathrm{H}_{2} \mathrm{O}-$ & 0.62 & - & - & 0.79 & 1.24 & - & - & - \\
\hline $\mathrm{CO}_{2}$ & 0.15 & - & - & 0.16 & 0.12 & - & - & - \\
\hline $\mathrm{Na}$ (ppm) & - & - & 2.21 & 2.14 & 2.02 & 2.01 & 2.06 & - \\
\hline K & - & - & 0.25 & 0.16 & 0.61 & 0.41 & 0.14 & - \\
\hline $\mathrm{K} / \mathrm{Na}$ & - & - & 0.11 & 0.075 & 0.30 & 0.20 & 0.06 & - \\
\hline $\mathrm{Li}$ & - & - & 16 & 10.5 & 7.52 & 9.81 & 5.44 & - \\
\hline $\mathrm{Rb}$ & - & - & 9.2 & 6.8 & 14.9 & 5.44 & 1.94 & - \\
\hline $\mathrm{K} / \mathrm{Rb}$ & - & - & 268 & 236 & 407 & 750 & 720 & - \\
\hline $\mathrm{Sr}$ & - & - & 183 & 171 & 490 & 445 & 121 & - \\
\hline B & - & - & $<11$ & $<10$ & $<10$ & - & - & - \\
\hline $\mathrm{F}$ & - & - & 255 & 244 & 488 & - & - & - \\
\hline $\mathrm{Cr}$ & - & - & 207 & 271 & 66 & 260 & 291 & - \\
\hline V & - & - & 277 & 364 & 272 & 316 & 286 & - \\
\hline $\mathrm{Cu}$ & - & - & 74 & 61 & 119 & 56 & 79 & - \\
\hline $\mathrm{Ni}$ & - & - & 73 & 92 & 56 & 128 & 123 & - \\
\hline Co & - & - & 67 & 50 & 46 & 46.4 & 43.7 & - \\
\hline $\mathrm{Zn}$ & - & - & 90 & 112 & 151 & 101 & 91 & - \\
\hline $\mathrm{U}$ & - & - & $0.2-0.7$ & $<0.1$ & $0.3-0.7$ & 0.35 & 0.28 & - \\
\hline Th & - & - & 0.3 & 0.3 & 1.9 & 0.21 & - & - \\
\hline $\mathrm{Cr} / \mathrm{Ni}$ & - & - & 2.4 & 3.0 & 1.2 & 2.0 & 2.4 & - \\
\hline $\mathrm{Ti} / \mathrm{Cr}$ & - & - & 80 & 50 & 620 & 60 & 50 & - \\
\hline
\end{tabular}

types originated. By parent magma, we mean the magma which has given rise to a series of igneous rocks, whether or not it was primitive or underwent a certain evolution.
To a larger extent, this may be applied to the Hole 446A basalts which are all characterized by a high iron content. Their distinctive trend shows no relationship with the Hole 442B and Hole 443 basalts. We think that 


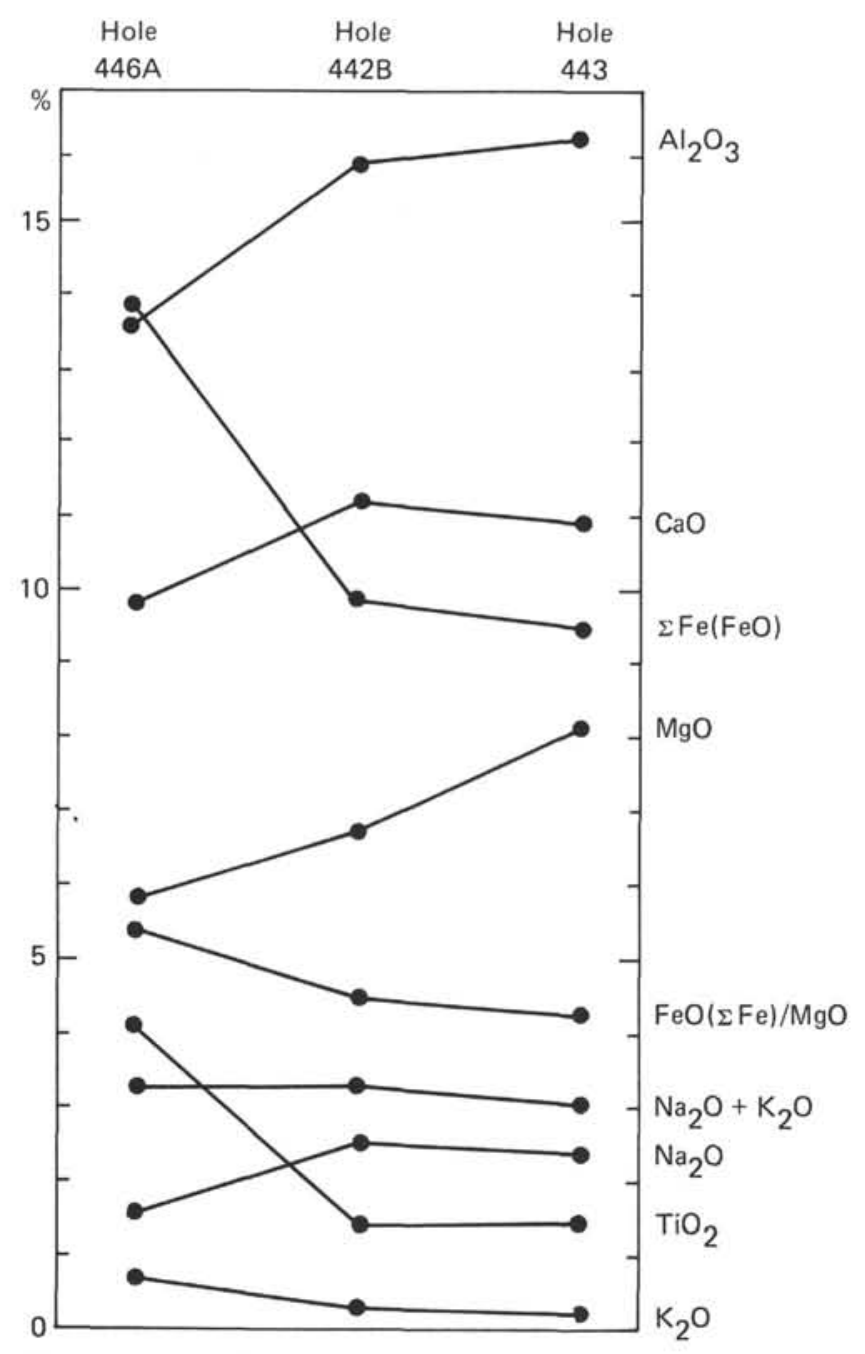

Figure 6. Variations among some Leg 58 basalts.

this conclusion is correct, because the presence of thick sills of similar composition at Site 446 , in the complete absence of oceanic basalts, may indicate a parent magma already enriched in iron and titanium. Magma evolution has given rise to the whole range of Site 446 ferrobasalts and alkaline rock varieties (Table 2; Figure 7).

In Figure 7, one may notice an interesting fact: the Site 446 basalts have the same differentiation index as the Hole 442B basalts, but a higher magnesium content. In other words, despite the high content of iron and titanium (suggesting extensive magma differentiation, in which a great decrease in magnesium content is common), the rocks under discussion have a higher magnesium content. This may point to a higher magnesium content in the parent magma (and to a considerable depth of its formation) which, in the process of magma evolution, has given rise to rocks described as ferrobasalts. This is in agreement with conclusions at which Bass (1971) and Scheidegger (1973) have arrived.

Ferrobasalts are comparatively widespread on a global scale. They are encountered in the eastern Indian ocean (Hekinian, 1974; Thompson et al., 1974; Robinson and Whitford, 1974), in the central and eastern
Pacific ocean, within the Juan de Fuca submarine ridge (Jackson et al., 1976; Clague and Bunch, 1976). Similar rock types are described by Sigursson et al. (1968) from Iceland. Such rock types also occur within continents in plateau basalts, both as independent intrusions of dolerites and as final products of their differentiation, exemplified by ferrogabbro of the Skaergaard intrusion, described in detail by Wager (1968).

Bass (1971) and Scheidegger (1973) attribute ferrobasalt to a considerable depth of magma formation. However, Clague and Bunch (1976) consider these rock types to be the result of intensive subsurface magma differentiation. Generally, ferrobasalt formation resembles the in-chamber differentiation hypothesized by Wager (1968) for the Skaergaard intrusion and supported by experimental data obtained by Kennedy (1955) and Osborn (1959), who showed that magma evolution resulting in iron accumulation in the final products of differentiation should proceed under a constant bulk composition of the dry melt (constant oxygen content). A high water content should result in a calc-alkaline trend of differentiation, with silica accumulation. Hamilton and Anderson (1967) arrived at the conclusion that water content in magma does not greatly influence magma differentiation.

The last conclusion is of principal importance, because melts producing the Shikoku and Daito basalts were characterized by various (including high) water contents, which nevertheless ensured the tholeiitic trend of magma evolution, resulting in accumulation of iron and not silica at the end of the process.

\section{WATER CONTENT IN MAGMA}

Basalts of the Shikoku Basin and Daito Basin have different vesicularities; Site 442 basalts are most vesicular, with average vesicularity of 20 to 25 per cent in lava flows and 25 to 30 per cent in pillow basalts.

Hole 443 basalts have vesicularity values of 3 and 15 per cent (in places up to $30 \%$ ), for flows and pillow basalts respectively. The bulk of the Hole 446A basalts are characterized by a vesicularity of 0 to 10 per cent (3\% average); in five units with amphibole vesicularity amounts to 15 to 30 per cent. Visual determination of vesicularity is in agreement with the porosity of the rock (site reports, this volume).

The high vesicularity of the Hole 442B basalts is not a sign of near-surface effusion, as hypothesized for the Reykjanes basalts (Moore and Schilling, 1973). High vesicularity is also typical of some Hole 443 and Hole 446A basalts which definitely formed at depths exceeding 4000 meters. Rock density in all cases is a function of porosity, rather than depth. Therefore, the different vesicularities of rocks from one hole and the high vesicularity of Hole 442B basalts were predetermined by different water contents in the magmas. The melts may have contained relatively high water concentrations, which is indicated by amphibole in the most-vesicular Hole 446A basalts.

Assuming that the density of the water gaseous phase is $0.09 \mathrm{~g} / \mathrm{cm}^{3}$ (Vakulovitch, 1965) at $1000^{\circ} \mathrm{C}$ and 500 atm (at a depth of $5 \mathrm{~km}$ ), the concentration of free water 

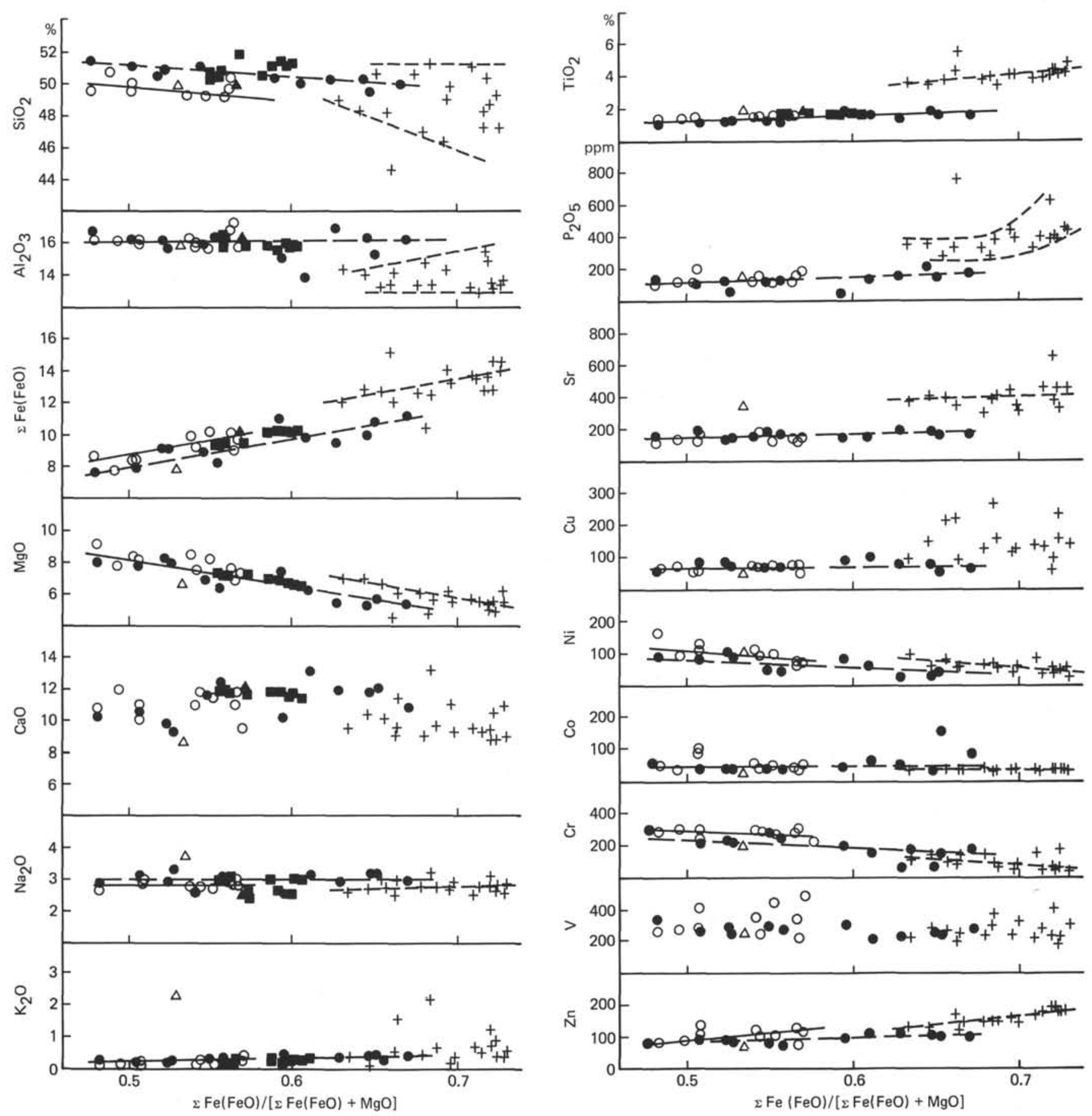

Figure 7. Behavior of major oxides and minor elements in the process of magma differentiation. $\Sigma \mathrm{Fe}(\mathrm{FeO}) /[\Sigma \mathrm{Fe}$ $(\mathrm{FeO})+\mathrm{MgOl}$ is an index of differentiation. Symbols are explained in Figure 1.

equals $0.27,1.35,2.25$, and 2.7 per cent at vesicularities of $3,15,25$, and 30 per cent, respectively. Noting the 0.3 per cent of water dissolved in basalts (Moore, 1970; Bryan and Moore, 1977), the total water content in the magma should be $0.6,1.6,2.5$, and 3.0 per cent. Therefore, minimum water content $(0.6 \%)$ is characteristic of the basalts of Hole 443 and the bulk of Hole $446 \mathrm{~A}$ basalts, while the maximum content $(2.5-3.0 \%)$ is determined for the Hole 442B basalts. Such high and inter- mediate water contents are also typical of the Hole 443 pillow basalts, as well as of the sills with amphibole in Hole 446A.

The data on rock chemical composition (Table 3) and the low vesicularity (relative dryness) of Hole 443 basalts enable us to attribute them to ridge basalts (Moore, 1970).

The high water content in the Hole 442 basalts with a tholeiitic trend of evolution apparently reflects the deci- 
sive influence of water upon magma differentiation (Hamilton and Anderson, 1967). This raises the question of the absence of amphibole in these rocks, as opposed to similar rocks in Hole 446 which contain amphibole. With due regard for experimental data (Yoder and Tilley, 1962; Lambert and Wyllie, 1972), this can be accounted for by high magma temperature (Hole 442B), outside the amphibole stability field. At the same time, the presence of amphibole in the Hole 446A basalts indicates either a lower magma temperature (compared with that for Hole 442B basalts) or a greater depth (higher pressure) of amphibole formation. The last interpretation is based on experimental data showing that amphibole is stable in a basaltic melt at a temperature somewhat lower than $1000^{\circ} \mathrm{C}$ and at 10 to $15 \mathrm{kbar}$ (Lambert and Wyllie, 1972). These data, as well as those obtained by Yoder and Tilley (1962), prove that amphibole crystallization in situ is impossible, because the stability field is above a pressure range of 1 to $1.5 \mathrm{kbar}$. This indicates that the amphibole was crystallized at depth, possibly in an intermediate chamber. Erupting onto the surface, such magma could produce rock with amphibole.

\section{RARE ELEMENTS IN THE SHIKOKU AND DAITO BASALTS}

The distribution and behavior of rare and minor elements in basalts are governed by regularities typical of basic magma (Table 2; Figure 7) which involve both initial composition of magma and differentiation peculiarities of the basalts in each hole of Leg 58 .

Generally, the behavior of minor elements in magma evolution is similar to in-chamber differentiation (Wager and Brown, 1968; Nisterenko and Almukhamedov, 1973). Typical of all the Leg 58 holes is an increase in rubidium, strontium, fluorine, and copper concentration (simultaneous with iron and alkali accumulation) and a decrease in chromium, nickel, and (to a lesser extent) cobalt concentration in the final products of differentiation. Such behavior of elements may be variously accounted for.

Some of the elements are closely associated with petrogenic elements, which predetermine the distribution and behavior of the minor elements. For instance, rubidium and strontium are closely associated with potassium, whose accumulation at the end of the process is accompanied by a gradual increase in minor elements (Figure 7). On the other hand, the distribution of nickel, and to some extent cobalt, is governed by magnesium. Yet, up to 20 per cent of the nickel in basalts can be found in sulfides, and up to 60 per cent of the cobalt in the titanomagnetite (Nisterenko and Almukhamedov, 1973; Nisterenko et al., 1974), presumably in the form of ferrites. The same can also be said of nickel and copper behavior, but only 10 to 20 per cent of these elements in the rock is associated with titanomagnetite.

Copper, with distinct chalcophile properties, occurs in sulfide form, primarily as chalcopyrite and partly as sulfide droplets (Vakhrushev, 1973; Czamanske and Moore, 1977). In the process of basalt alteration, copper is reduced to native copper, which has been iden- tified in many polished sections and is partly removed into carbonate veins (Sample 443-54-3, $108 \mathrm{~cm}$ ).

Chromium concentration gradually decreases as the final products of differentiation are formed. Minimum chromium content is typical of basalts with the highest iron content (Hole 446A). Such behavior is quite natural, because chromium is removed from the melt at early differentiation stages, chiefly in chrome spinels and partly by substitution in ore minerals and pyroxenes.

The behavior of vanadium is somewhat obscure. It is commonly associated with titanium, and the two elements should accumulate together toward the end of melt evolution. The process of in-chamber magma differentiation is, for instance, characterized by a direct correlation of the two elements and intensive $(>1$ wt. \%) vanadium accumulation in titanomagnetite (Nisterenko and Almukhamedov, 1973). In the Shikoku and Daito basalts, this behavior of vanadium is noted only in rocks from Holes 443 and $446 \mathrm{~A}$; in Hole 442B basalts, such behavior is not noted, which defies explanation.

Fluorine (along with phosphorus) gradually accumulates at the end of the magma differentiation process and is seemingly bound with phosphorus in apatite.

The behavior of other minor elements (lithium, boron, zinc, uranium, thorium, rare earths) in the process of magma evolution is either obscure or cannot be definitely traced because of lack of analyses.

On the whole, the behavior and distribution of minor elements in the process of the Shikoku and Daito basalt formation (Figure 7) support the conclusions on the role of major elements in this process and testify to distinct (Hole 446A) or slightly different (Holes 442B and 443) magma compositions and different intensities of magma differentiation.

\section{SUMMARY}

1. Basalts from the northern Philippine sea are of different compositions: in Hole 443, they correspond to mid-ocean-ridge basalts; in Hole 442B, to off-ridge basalts with a lower magnesium content; in Hole 446A, to ferrobasalts. These differences are predetermined by the various compositions of the parent magmas, which are slightly different for Sites 442 and 443 and substantially different for Site 446.

In normative composition, the basalts are olivine tholeiites (normative olivine and hypersthene, Holes 442B and 443) and saturated tholeiites (normative hypersthene, Site 446).

2. Off-ridge basalts from Hole $442 \mathrm{~B}$ have distinct compositions and require additional studies. However, the data obtained by Hart (1976) indicate that their lower magnesium content is typical for the world ocean.

3. Basalts from Hole 443 indicate minimum differentiation, those from Hole 442 considerable differentiation, and those from Site $\mathbf{4 4 6}$ maximum differentiation of parent magma. In all three cases, magma evolution, irrespective of water content, was of a tholeiitic trend, resulting in accumulation of iron, titanium, and alkalis. Melt differentiation was accompanied by fractionation 
of olivine, plagioclase, and (in Site 446 basalts) pyroxene.

4. All basalts of Hole 442B and the Hole 446A sills (with amphibole) are characterized by high (up to $30 \%$ ) vesicularity, suggesting maximum 3 per cent water content in the erupted magma. The absence of amphibole in Hole 442B basalts may be accounted for by higher magma temperature as compared with that for Site 446 basalts.

5. Site 446 basalts, notable for the highest iron content and intensive enrichment in titanium (ferrobasalts), are not a common rock type. These are, however, normal igneous rocks of oceanic origin. Such rocks occur in the eastern Indian Ocean, in the central and eastern Pacific Ocean, and within the Juan de Fuca ridge. Also, similar rocks are found in Iceland and within continents among plateau basalts and are described as the final products of differentiation of tholeiitic intrusions (ferrogabbros).

\section{REFERENCES}

Bass, M. N., 1971. Variable abyssal basalt populations and their relation to sea-floor spreading rates. Earth Planet. Sci. Lett., 11, 18-22.

Bryan, W. B., and Moore, J. G., 1977. Compositional variations of young basalts in the Mid-Atlantic Ridge rift valley near lat. $36^{\circ} 49^{\prime}$ N. Geol. Soc. Am. Bull., 88, 556-570.

Clague, D. A., and Bunch, T. L., 1976. Formation of ferrobasalt at east Pacific midocean spreading centers. J. Geophys. Res., 81, 4247-4268.

Czamanske, G. K., and Moore, J. G., 1977. Composition and phase chemistry of sulfide globules in basalt from the MidAtlantic Ridge rift valley near $37^{\circ}$ lat. Geol. Soc. Am. Bull., 88, 587-599.

Dmitriev, L. V., Sharas'kin, A. Ja., and Garanin, A. V., 1976. The main features of magmatism within the ocean floor. In Proceedings of the V All-Union Petrographic Conference: Moscow (Nauka).

Frey, F. A., Bryan, W. B., and Thompson, G., 1974. Atlantic Ocean floor: geochemistry and petrology of basalts from Legs 2 and 3 of the Deep Sea Drilling Project. J. Geophys. Res., 79, 5507-5528.

Hamilton, D. L., and Anderson, G. M., 1967. Effects of water and oxygen pressures on the crystallization of basaltic magmas. In Poldervaart, R. (Ed.), Basalts, (vol. 1), 445482.

Hart, S., 1976. Chemical variance in deep ocean basalts. In Hart, S., Yeats, R., et al., Init. Repts. DSDP, 34: Washington (U. S. Govt. Printing Office), pp. 301-331.

Hekinian, R., 1974. Petrology of igneous rocks from Leg 22 in the northeastern Indian Ocean. In von der Borch, C., Sclater, J., et al., Init. Repts. DSDP, 22: Washington (U. S. Govt. Printing Office), pp. 413-448.
Irvine, T. N., and Baragar, W. R. A., 1971. A guide to chemical classification of the common volcanic rocks. Can. J. Earth Sci., 8, 523-548.

Jackson, E. D., Bargar, K. E., Fabbi, B. P., and Herapoulos, C., 1976. Petrology of the basaltic rocks drilled on Leg 33 of the Deep Sea Drilling Project. In Jackson, E., Schlanger, S., et al., Init. Repts. DSDP 33: (Washington (U. S. Govt. Printing Office), pp. 571-630.

Kennedy, G. C., 1955. Some aspects of the role of water in rock melts. Geol. Sci. Am. Special Paper, 62, 489-504.

Klein, G. deV. Kobayashi, K., Chamley, H., Curtis, D. M., Dick, U. J. B., Echols, D. J., Fountain, D. M., Kinoshita, H., Marsh, N. G., Nisterenko, G. V., Okada, H., Sloan, J. K., Waples, D. M. and White, S. M., 1978. Off-ridge volcanism and sea floor spreading in the Shikoku Basin. Nature, 273, 746-748.

Lambert, J. B., Wyllie, P. J., 1972. Melting of gabbro (quartz eclogite) with excess water to 35 kilobars, with geological applications. J. Geol., 80, 693-708.

Moore, J. G., 1970. Water content of basalt erupted on the ocean floor. Contr. Mineral. Petrol., 28, 272-279.

Moore, J. G., and Schilling, J.-G., 1973. Vesicles, water and sulfur in Reykjanes ridge basalts. Contr. Mineral. Petrol., $41,105-118$

Nisterenko, G. V., and Almukhamedov, A. I., 1973. Geochemistry of Differentiated Dolerites: Moscow (Nauka).

Nisterenko, G. V., Laktionova, N. V., and Medvedeva, L. S., 1974. On the role of sulfide form of copper (nickel, cobalt) in andesite-basalts of Chile. Geokhimiya, No. 1, 89-94.

Osborn, E. F., 1959. Role of oxygen pressure in the crystallization and differentiation of basaltic magma. Geokhimiya, 257, 609-647.

Robinson, P. T., and Whitford, D. J., 1974. Basalts from the eastern Indian Ocean, DSDP Leg 27. In Heirtzler, J., Veevers, J. et al. Init. Repts. DSDP, 27: Washington (U. S. Govt. Printing Office), pp. 551-559.

Scheidegger, K. F., 1973. Temperatures and compositions of magmas ascending along midocean ridges. J. Geophys. Res., 78, 3340-3355.

Sigursson, H., Schilling, J.-G., and Meyer, P. S., 1978. Svagi and Langjökull volcanic zones in Iceland: petrology and structure. J. Geophys. Res., 83, 3971-3982.

Thompson, G., Bryan, W. B., Frey, F. A., and Sung, C. M., 1974. Petrology and geochemistry of basalts and related rocks from Sites 214, 215, 216, DSDP Leg 22, Indian Ocean. In von der Borch, C., Sclater, J., et al, Init. Repts. DSDP, 22: Washington (U. S. Govt. Printing Office), pp. 459-468.

Vakulovitch, M. P., 1965. Tables Showing Thermodynamic Properties of Water and Water Vapor: Moscow (Energy).

Vakhrushev, V. A., 1973. Ore Minerals in Igneous Rocks and Their Significance in Petrographic Studies: Novosibirsk (Nauka).

Wager, L., and Brown, G., 1968. Layered Igneous Rocks: London (Oliver-Road).

Yoder, H. S., Jr., and Tilley, C. E., 1962. Origin of basalt magmas. J. Petrol., 3, 473-575. 


\section{APPENDIX}

Chemical Composition of Unrepresentative Basalts (with high carbon-dioxide concentration or extensive oxidation)

\begin{tabular}{|c|c|c|c|c|c|c|c|c|c|c|c|c|c|c|c|}
\hline $\begin{array}{c}\text { Sample } \\
\text { (interval in } \mathrm{cm} \text { ) }\end{array}$ & $\mathrm{SiO}_{2}$ & $\mathrm{TiO}_{2}$ & $\mathrm{Al}_{2} \mathrm{O}_{3}$ & $\mathrm{Fe}_{2} \mathrm{O}_{3}$ & $\mathrm{FeO}$ & $\mathrm{MnO}$ & $\mathrm{MgO}$ & $\mathrm{CaO}$ & $\mathrm{Na}_{2} \mathrm{O}$ & $\mathrm{K}_{2} \mathrm{O}$ & $\mathrm{P}_{2} \mathrm{O}_{5}$ & $\mathrm{H}_{2} \mathrm{O}+$ & $\mathrm{H}_{2} \mathrm{O}-$ & $\mathrm{CO}_{2}$ & Total \\
\hline $442 A-31-1,81-86^{a}$ & 49.40 & 1.43 & 15.55 & \multicolumn{2}{|c|}{8.80} & 0.16 & 6.74 & 13.42 & 2.94 & 0.31 & .141 & 0.47 & - & 0.22 & 99.59 \\
\hline $32-1,50-55^{a}$ & 49.60 & 1.42 & 15.30 & \multicolumn{2}{|c|}{8.88} & 0.15 & 6.55 & 12.85 & 2.92 & 0.32 & .128 & 0.78 & - & 0.32 & 99.30 \\
\hline $32-2,101-106^{a}$ & 48.50 & 1.36 & 14.65 & \multirow{2}{*}{\multicolumn{2}{|c|}{$\begin{array}{l}8.87 \\
8.65\end{array}$}} & 0.13 & 6.41 & 13.41 & 2.94 & 0.28 & .132 & 1.67 & - & 0.67 & 99.14 \\
\hline $33-1,62-66^{a}$ & 48.40 & 1.24 & 16.30 & & & 0.15 & 7.00 & 13.28 & 2.76 & 0.32 & .137 & 1.12 & - & 2.32 & 101.74 \\
\hline $33-1,118-122^{a}$ & 47.50 & 1.28 & 15.60 & \multicolumn{2}{|c|}{8.57} & 0.15 & 6.23 & 14.84 & 2.66 & 0.24 & .137 & 0.65 & - & 1.85 & 99.70 \\
\hline $33-3,81-87 a$ & 49.50 & 1.31 & 15.32 & \multirow{2}{*}{\multicolumn{2}{|c|}{$\begin{array}{l}8.93 \\
8.82\end{array}$}} & 0.13 & 6.39 & 14.41 & 2.71 & 0.24 & .121 & 1.17 & - & 1.21 & 101.48 \\
\hline $33-4,83-88^{a}$ & 49.20 & 1.38 & 14.79 & & & 0.14 & 6.75 & 13.07 & 2.95 & 0.40 & .114 & 1.79 & - & 0.85 & 100.34 \\
\hline $34-1,5-10^{a}$ & 49.10 & 1.33 & 15.52 & \multicolumn{2}{|c|}{8.85} & 0.13 & 5.78 & 13.01 & 2.77 & 0.26 & .146 & 0.97 & - & 0.39 & 98.26 \\
\hline $34-1,110-118^{a}$ & 49.50 & 1.32 & 15.24 & \multicolumn{2}{|c|}{8.44} & 0.16 & 6.21 & 13.47 & 2.99 & 0.42 & .107 & 1.98 & - & 1.10 & 100.98 \\
\hline $442 \mathrm{~B}-3-3,30-36^{\mathrm{a}}$ & 49.70 & 1.46 & 16.02 & \multicolumn{2}{|l|}{8.32} & 0.16 & 6.22 & 11.80 & 2.80 & 0.27 & .141 & 2.81 & - & 1.50 & 101.22 \\
\hline $3-4,26-30^{\mathrm{a}}$ & 49.20 & 1.37 & 14.80 & \multicolumn{2}{|c|}{8.66} & 0.15 & 6.18 & 16.02 & 2.85 & 0.23 & .093 & 0.46 & - & 0.05 & 100.11 \\
\hline $3-4,64-70^{b}$ & 47.20 & 1.28 & 15.45 & 4.89 & 3.91 & 0.14 & 6.42 & 13.71 & 2.74 & 0.25 & .141 & 1.42 & 0.56 & 2.17 & 100.23 \\
\hline $5-2,83-88^{a}$ & 48.90 & 1.29 & 15.15 & \multicolumn{2}{|c|}{9.06} & 0.15 & 8.43 & 10.81 & 2.95 & 0.18 & .123 & 2.58 & - & 0.55 & 100.23 \\
\hline $6-2,25-29$ & 48.90 & 1.25 & 15.40 & 5.51 & 3.79 & 0.14 & 7.47 & 11.69 & 2.72 & 0.36 & .119 & 1.19 & 0.80 & 0.61 & 100.10 \\
\hline $9-2,120-125^{a}$ & 48.80 & 1.22 & 15.25 & \multicolumn{2}{|c|}{9.07} & 0.14 & 6.58 & 12.13 & 2.86 & 0.31 & .054 & 3.00 & - & 1.01 & 100.42 \\
\hline $12-1,95-100^{b}$ & 48.80 & 1.68 & 16.72 & \multirow{2}{*}{\multicolumn{2}{|c|}{$\begin{array}{r}10.53 \\
8.06\end{array}$}} & 0.23 & 3.87 & 11.52 & 3.16 & 0.73 & .114 & 2.35 & - & 0.32 & 100.02 \\
\hline $16-2,20-28^{b}$ & 49.80 & 1.29 & 16.46 & & & 0.11 & 6.71 & 12.13 & 2.85 & 0.43 & .137 & 1.39 & - & 0.03 & 99.42 \\
\hline $443-50-2,130-135$ & 49.00 & 1.71 & 17.05 & 3.48 & 6.41 & 0.25 & 5.35 & 11.50 & 2.98 & 0.17 & .132 & 1.00 & 0.25 & 0.72 & 100.00 \\
\hline $57-1,71-75$ & 44.20 & 1.57 & 13.25 & 5.21 & 5.30 & 0.19 & 6.65 & 14.10 & 2.43 & 0.43 & .155 & 1.42 & 0.83 & 4.21 & 99.94 \\
\hline $57-2,114-118$ & 47.10 & 1.82 & 14.60 & 6.34 & 5.55 & 0.22 & 5.86 & 11.70 & 2.88 & 0.56 & .229 & 0.79 & 0.95 & 1.03 & 99.63 \\
\hline $446 \mathrm{~A}-12-4,47-53$ & 44.70 & 3.60 & 10.90 & 5.27 & 5.98 & 0.22 & 10.20 & 11.75 & 2.07 & 0.85 & .389 & & 17 & 1.32 & 99.50 \\
\hline $13-1,17-23$ & 42.50 & 3.35 & 13.30 & 5.64 & 5.81 & 0.22 & 6.86 & 14.10 & 2.51 & 1.22 & .412 & & 48 & 2.30 & 99.70 \\
\hline
\end{tabular}

${ }_{\mathrm{b}}^{\mathrm{a}} \mathrm{X}$-Ray-fluorescence method.

${ }^{b}$ Strongly oxidized basalts. 